\title{
On Eddy Viscosity, Energy Cascades, and the Horizontal Resolution of Gridded Satellite Altimeter Products*
}

\author{
BRIAN K. ARBIC \\ Department of Earth and Environmental Sciences, University of Michigan, Ann Arbor, Michigan
}

KURT L. POLZIN

Physical Oceanography Department, Woods Hole Oceanographic Institution, Woods Hole, Massachusetts

ROBERT B. SCOTT

Institute for Geophysics, Jackson School of Geosciences, The University of Texas at Austin, Austin, Texas, and Departement de Physique et LPO, Université de Bretagne Occidental, CNRS, Brest, France

JAMES G. RICHMAN AND JAY F. SHRIVER

Oceanography Division, Naval Research Laboratory, Stennis Space Center, Mississippi

(Manuscript received 21 December 2011, in final form 23 August 2012)

\begin{abstract}
Motivated by the recent interest in ocean energetics, the widespread use of horizontal eddy viscosity in models, and the promise of high horizontal resolution data from the planned wide-swath satellite altimeter, this paper explores the impacts of horizontal eddy viscosity and horizontal grid resolution on geostrophic turbulence, with a particular focus on spectral kinetic energy fluxes $\Pi(K)$ computed in the isotropic wavenumber $(K)$ domain. The paper utilizes idealized two-layer quasigeostrophic $(\mathrm{QG})$ models, realistic highresolution ocean general circulation models, and present-generation gridded satellite altimeter data. Adding horizontal eddy viscosity to the QG model results in a forward cascade at smaller scales, in apparent agreement with results from present-generation altimetry. Eddy viscosity is taken to roughly represent coupling of mesoscale eddies to internal waves or to submesoscale eddies. Filtering the output of either the QG or realistic models before computing $\Pi(K)$ also greatly increases the forward cascade. Such filtering mimics the smoothing inherent in the construction of present-generation gridded altimeter data. It is therefore difficult to say whether the forward cascades seen in present-generation altimeter data are due to real physics (represented here by eddy viscosity) or to insufficient horizontal resolution. The inverse cascade at larger scales remains in the models even after filtering, suggesting that its existence in the models and in altimeter data is robust. However, the magnitude of the inverse cascade is affected by filtering, suggesting that the wide-swath altimeter will allow a more accurate determination of the inverse cascade at larger scales as well as providing important constraints on smaller-scale dynamics.
\end{abstract}

* Naval Research Laboratory Contribution Number NRL/JA/ 7320-2011-802, and The University of Texas at Austin Institute for Geophysics Contribution Number 2482.

Corresponding author address: Dr. Brian K. Arbic, Department of Earth and Environmental Sciences, University of Michigan, Ann Arbor, MI 48109-1005.

E-mail: arbic@umich.edu

\section{Introduction}

This paper examines the impacts of horizontal eddy viscosity and of horizontal grid resolution on geostrophic turbulence. Particular emphasis is placed on spectral fluxes $\Pi(K)$ of surface ocean geostrophic kinetic energy computed in the isotropic wavenumber $(K)$ domain from an idealized two-layer quasigeostrophic (QG) turbulence model, from a high-resolution ocean model run in 
a realistic near-global domain (hereafter, the "realistic" model), and from present-generation gridded satellite altimeter data. We are motivated by considerations of ocean energetics and by the widespread use of horizontal eddy viscosities in models. We are especially interested in whether forward kinetic energy cascades may represent a significant sink of energy in the ocean and may be triggered by processes that can be roughly characterized by a horizontal eddy viscosity. The likelihood of greatly increased horizontal resolution in planned future-generation wide-swath satellite altimeter data (Fu and Ferrari 2008; $\mathrm{Fu}$ et al. 2012) motivates us to examine here the importance of horizontal grid resolution in the estimation of spectral fluxes, particularly at smaller scales. An additional goal of the paper is to directly compare the inverse cascades at larger scales diagnosed in spectral fluxes $\Pi(K)$ in realistic models with the inverse cascades seen in present-generation gridded altimeter data, as a test of the robustness of both. The model-data comparison will be conducted over six oceanic regions, which vary widely in their eddy kinetic energy levels.

Recently the oceanographic community has focused considerable attention on quantifying the mechanisms and spatial distribution of energy input, energy dissipation, and eddy diffusivity in the ocean. The recent interest in ocean energetics has arisen in part because ocean mixing is thought to exert a strong control on abyssal stratification and circulation (e.g., Munk and Wunsch 1998). One important energy source is the $\sim 1$-TW wind power input into geostrophic flows (e.g., Wunsch 1998; Scott 1999; Scott and Xu 2009). Several recent papers have explored potential dissipation mechanisms for this energy. Sen et al. (2008), Arbic et al. (2009), Wright et al. (2013), and references therein argue that quadratic bottom boundary layer drag is an important energy sink for low-frequency flows. Marshall and Naveira-Garabato (2008), Nikurashin and Ferrari (2011), and Scott et al. (2011) argue that internal lee waves generated by geostrophic flows over rough topography are a significant energy sink. An additional mechanism for dissipation over rough topography has recently been proposed by Dewar and Hogg (2010).

Here we consider another potential mechanism, namely, a forward cascade of kinetic energy to small scales, particularly in the upper 1000 or so meters of the ocean (i.e., the thermocline). In the models used in this paper, the forward kinetic energy cascade will be triggered and dissipated by horizontal eddy viscosity $\nu$ (hereafter, "viscosity"). ${ }^{1}$ Viscosity has long been employed in numerical ocean

\footnotetext{
${ }^{1}$ The important subject of vertical eddy viscosity will not be addressed in this paper.
}

models (e.g., Müller 1979, among many). Viscosity is used to absorb subgrid-scale structure in models and is generally kept as small as the model resolution allows (e.g., Wallcraft et al. 2005). The effects of viscosity on ocean climate models have been investigated by Jochum et al. (2008). To our knowledge, this paper presents the first study of the impacts of viscosity on the statistical properties of eddies, including spectral kinetic energy fluxes $\Pi(K)$, in idealized QG turbulence models. Although the focus of the QG sensitivity study is on the spectral kinetic energy fluxes $\Pi(K)$, we will also briefly discuss the sensitivity of the energy levels, horizontal scales, and vertical structure of eddies in the QG turbulence model to viscosity. We will examine the impact of viscosity on the energy budget of the QG model. The sensitivity study to viscosity presented here serves as a complement to our earlier studies of the sensitivity of QG turbulence to linear bottom drag (Arbic and Flierl 2004; Arbic et al. 2007; see also Thompson and Young 2006, 2007). See also Arbic and Scott (2008) for an examination of the sensitivity of QG turbulence to quadratic bottom drag. In addition, we will examine the impact of viscosity on the spectral kinetic energy fluxes $\Pi(K)$ computed from realistic models. We emphasize again that, for the sake of simplicity, we focus here on the spectral fluxes of geostrophic kinetic energy. Any ageostrophic motions that may be present in the realistic model will be removed, at least roughly, with a low-pass filter in the time domain.

The spectral fluxes $\Pi(K)$ reveal the direction of the nonlinear energy cascade and were computed from present-generation gridded satellite altimeter data by Scott and Wang (2005), following similar pioneering calculations performed on atmospheric data by Boer and Shepherd (1983). Scott and Arbic (2007) examined spectral kinetic energy fluxes $\Pi(K)$ in the baroclinic mode, barotropic mode, and upper layer of a two-layer QG model and used the model results to interpret the inverse cascade of kinetic energy seen in altimetry data by Scott and Wang (2005) at larger scales. Scott and Arbic (2007) did not explore the forward cascade seen in altimetry data at smaller scales. Here we focus much more attention on this forward cascade. One hypothesis explored in this paper is that the forward cascade seen in present-generation altimeter data is consistent with the action of processes that can be roughly characterized by a horizontal eddy viscosity.

We will not discuss in great detail here the mechanisms that may lie behind an oceanic forward kinetic energy cascade. However, a few brief remarks are in order. Capet et al. (2008) demonstrated that frontogenesis in regions of active submesoscale eddies yields a forward kinetic energy cascade that drains energy from the mesoscale. The forward cascade in the Capet et al. (2008) results is driven fundamentally by ageostrophic 
velocities. In support of the frontogenesis mechanism, D'Asaro et al. (2011) presented observational evidence of enhanced energy dissipation in ocean fronts. Another postulated mechanism for a forward cascade is interactions between mesoscale eddies and the internal wave field. Building upon studies of wave-mean flow interactions (Müller and Olbers 1975; Müller 1976; Bühler and McIntyre 2005), Polzin $(2008,2010)$ argued that mesoscale eddy-internal wave interactions can be parameterized by a viscosity. Based on an updated analysis of Polygon Mid-Ocean Dynamics Experiment (POLYMODE) data, Polzin $(2008,2010)$ finds comparable, but somewhat smaller, estimates of viscosities than Brown and Owens (1981) obtained from the same dataset.

We will also not contribute here to recent discussions (e.g., LaCasce 2012 and references therein) about whether the altimeter signal consists primarily of the first baroclinic mode of an "interior QG" solution (Wunsch 1997), or of a "surface quasi-geostrophic" (SQG) solution (Lapeyre 2009). The idealized two-layer QG model used in our study does not include any SQG dynamics, and the realistic model employed here does not have enough vertical resolution to properly include SQG effects. Our choice of models is made based on what is easily available to us, and not because we believe SQG dynamics to be unimportant.

Motivated by the relatively coarse resolution of present-day gridded satellite altimeter data, this paper addresses the impact of horizontal grid resolution on the estimation of spectral kinetic energy fluxes. Along-track altimeter data, which is one-dimensional, cannot be used for spectral flux computations, which require a twodimensional field. Instead, observationally based computations of spectral fluxes must rely on two-dimensional gridded altimeter products. The Archiving, Validation, and Interpretation of Satellite Oceanographic (AVISO) gridded altimeter data (Le Traon et al. 1998; Ducet et al. 2000) used by Scott and Wang (2005) to compute spectral kinetic energy fluxes are constructed by interpolating along-track data from two satellite altimeters, in space and time, onto a $1 / 3^{\circ}$ Mercator grid, with 7 -day spacing in time. The Ocean Topography Experiment (TOPEX)/ Poseidon and Jason altimeters utilized in this procedure have high along-track resolution (about $6 \mathrm{~km}$ ), but a large distance between adjacent tracks and a long sampling period (about $300 \mathrm{~km}$ and 9.9 days, respectively; Chelton et al. 2001). Because of the large cross-track distance and long sampling period, the feature resolution of gridded AVISO data is much coarser than $6 \mathrm{~km}$ (Chelton et al. 2011). The interpolation procedure used to construct gridded AVISO data acts to smooth the raw along-track data, in both space and time. Motivated by this smoothing, we examine here spectral kinetic energy fluxes $\Pi(K)$ estimated from filtered versus unfiltered output of our models.

Capet et al. (2008) noted that the zero crossings (wavenumbers at which the spectral kinetic energy fluxes $\Pi(K)$ cross zero, i.e., change sign from negative to positive) were rather different in their simulations compared to the Scott and Wang (2005) altimetrically-derived fluxes. Capet et al. (2008) noted that "it is unclear if the forward cascade present in these [altimeter] observations occurs for the same reasons than in our [the Capet et al. (2008)] simulations." Our study of the effects of smoothing on $\Pi(K)$ estimates will shed some light on whether these discrepancies may be due at least in part to sampling artifacts. In effect, then, our alternative hypothesis for the forward cascades seen in present-generation altimeter data is that they are due to smoothing in the AVISO data. In this exercise we are also motivated by Boer and Shepherd (1983), who discussed the possibility that the forward cascades they saw at small scales were artifacts of the resolution limitations in the atmospheric reanalyses they used.

The planned wide-swath altimeter will have repeat periods comparable to those of TOPEX/Jason; 22 days has been discussed as a target, for example ( $\mathrm{Fu}$ et al. 2012). As we will show, temporal smoothing can distort the spectral fluxes $\Pi(K)$ of geostrophic kinetic energy computed from the sea surface height (SSH) field. However, because snapshots of wide-swath data will be twodimensional (and at high horizontal resolution), it will be possible to compute spectral fluxes $\Pi(K)$ directly from the snapshots. Since the spatial and temporal smoothing needed to construct current-generation gridded altimeter datasets will be much reduced, spectral fluxes $\Pi(K)$ computed from wide-swath altimetry should be much more reliable. Prompted by a reviewer, we emphasize here that altimetry, even high-resolution wide-swath altimetry, can only be used to compute spectral fluxes $\Pi(K)$ arising from geostrophic velocities. Spectral fluxes arising from ageostrophic effects can be computed from models (e.g., Capet et al. 2008), whose validity at smaller scales can be checked by comparison of the model SSH fields with the wide-swath altimeter data.

We will compare spectral fluxes $\Pi(K)$ computed from fully resolved outputs of the idealized QG model and of the realistic model, with spectral fluxes computed from spatially- and/or temporally filtered versions of these outputs. The fluxes computed from filtered versions of the models are taken to roughly represent the state-ofthe-art in regards to what can be done with present-day, somewhat coarse-resolution gridded altimeter products. The fluxes computed from unfiltered model output are taken to roughly represent what could be done with the proposed wide-swath satellite altimeter. We will focus 
on the impact of horizontal resolution on the estimation of the forward cascade at small scales, but will examine resolution effects on the estimated inverse cascade at larger scales as well.

The paper is organized as follows. Section 2 orients the reader by presenting a typical spectral flux profile computed from present-generation gridded altimeter data. Section 3 presents a description of the QG turbulence model, a discussion of the impacts of viscosity on the QG model behaviors, and a discussion of the impact of both spatial and temporal filtering on the spectral fluxes $\Pi(K)$ computed from the QG model. Section 4 presents spectral fluxes $\Pi(K)$ computed from unfiltered and filtered output of the realistic model, alongside fluxes computed from present-generation gridded satellite altimeter (AVISO) data. A summary and discussion are presented in section 5 .

\section{Example spectral flux curve from gridded altimeter data}

To set the stage for discussions to follow, in Fig. 1 we display the spectral fluxes $\Pi(K)$ computed over the Kuroshio region from AVISO gridded altimeter data. Details of the AVISO $\Pi(K)$ calculations will be discussed later, in section 4 . Figure 1 illustrates the nearly universal shape seen in the spectral kinetic energy fluxes $\Pi(K)$ computed throughout the Pacific Ocean by Scott and Wang (2005). A positive lobe at smaller scales

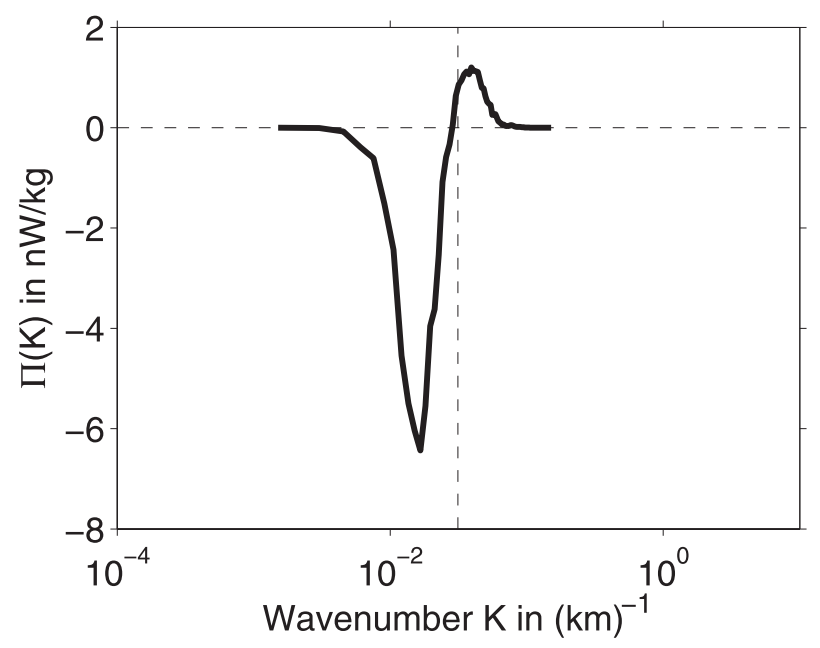

FIG. 1. Spectral fluxes $\Pi(K)$ of surface ocean geostrophic kinetic energy vs isotropic wavenumber $K$ in Kuroshio region, computed from AVISO gridded satellite altimeter data as described later in section 4. Dashed vertical line denotes deformation wavenumber $1 / L_{d}$ for this region. The data source for the $L_{d}$ value is also described later in section 4 .

(larger wavenumbers) indicates a forward, or direct, cascade of energy toward yet smaller scales. A larger negative lobe at larger scales indicates an inverse cascade of kinetic energy toward yet larger scales. The relative size of the forward and inverse cascades can be measured by the quantity FtoI, which we define by

$$
\text { FtoI }=-\frac{\text { maximum positive value of } \Pi(K) \text { at smaller scales }}{\text { minimum negative value of } \Pi(K) \text { at larger scales }} .
$$

For the $\Pi(K)$ curve in Fig. 1, the FtoI value is 0.19, which lies in the middle of the FtoI values computed later in section 4 over six different oceanic regions. The deformation wavenumber $1 / L_{d}$, where $L_{d}$ is the first baroclinic mode deformation radius, is shown as a dashed vertical line in Fig. 1 . The data source for the oceanic $L_{d}$ values used in Fig. 1 and elsewhere in the paper will also be described in section 4. For the $\Pi(K)$ curve shown in Fig. 1, the deformation wavenumber lies at the zero crossingthe transition between the negative and positive lobes. Later we will see that the transition wavenumber in the models we examine is higher than $1 / L_{d}$ unless the models employ relatively large horizontal eddy viscosity.

\section{The idealized quasigeostrophic model}

\section{a. Description of idealized model}

We use the same idealized two-layer QG turbulence model as in many of our previous papers (e.g., Arbic and
Flierl 2004; see also Flierl 1978). The model is forced by an imposed mean flow, which is taken to roughly represent the time-mean flows of a subtropical gyre. The mean flow is horizontally homogeneous within each layer. The model is doubly periodic, is run here on an $f$ plane with a flat bottom for simplicity, and has 256 gridpoints on a side (thus $256^{2}$ gridpoints in all), where each side has dimensional length $20 \pi L_{d}$ except where noted. The mean flow is vertically sheared between the layers and is therefore baroclinically unstable. The model is initialized with a randomly generated initial condition and achieves statistical equilibrium when the energy extracted by eddies from the baroclinically unstable mean flow is balanced by energy dissipation. For this paper, in contrast to our earlier papers on QG turbulence, we include a viscosity in most of our simulations.

The quantity in our two-layer QG model that most closely corresponds to altimeter measurements of SSH is the upper-layer streamfunction. Therefore, we focus 
the majority of our attention in this paper on a layer interpretation of the two-layer QG model. The governing equations of the model in layer form are

$$
\begin{aligned}
& \frac{\partial q_{1}}{\partial t}+\Gamma_{1}+J\left(\psi_{1}, q_{1}\right)=w f+\nu \nabla^{4} \psi_{1}, \quad \text { and } \\
& \frac{\partial q_{2}}{\partial t}+\Gamma_{2}+J\left(\psi_{2}, q_{2}\right)=-R_{2} \nabla^{2} \psi_{2}+w f+\nu \nabla^{4} \psi_{2}
\end{aligned}
$$

where $t$ is time, potential vorticity $q$ is the prognostic variable, and the subscripts 1 and 2 denote the upper and lower layers, respectively. The zonal (east-west) and meridional (north-south) spatial coordinates are respectively $x$ and $y$, and $J(A, B)=\partial A / \partial x \partial B / \partial y-\partial A /$ $\partial y \partial B / \partial x$. Fluctuations from the time mean are written without overbars, while imposed time-mean quantities are designated with overbars. The forcing terms are

$$
\begin{aligned}
& \Gamma_{1}=\overline{u_{1}} \frac{\partial q_{1}}{\partial x}+\frac{\partial \overline{q_{1}}}{\partial y} \frac{\partial \psi_{1}}{\partial x}, \text { and } \\
& \Gamma_{2}=\overline{u_{2}} \frac{\partial q_{2}}{\partial x}+\frac{\partial \overline{q_{2}}}{\partial y} \frac{\partial \psi_{2}}{\partial x} .
\end{aligned}
$$

The zonal velocity is $u$, the meridional velocity is $v$, and the imposed time-mean flow is taken to be zonal. A wavenumber filter used for subgrid scale dissipation is denoted by $w f$. As shown by LaCasce (1996), the wavenumber filter does not distort modons (Stern 1975; Larichev and Reznik 1976a,b), which are exact solutions of the inviscid nonlinear equations, nearly as much as horizontal eddy viscosities or hyperviscosities do. This suggests that the wavenumber filter is a very "clean" choice for subgrid-scale dissipation in a model. As discussed in Arbic and Flierl (2004), the wavenumber filter has little effect (less than $3 \%$ ) on the energy budgets of the two-layer forced-dissipated QG turbulence simulations in that paper. This further demonstrates that the wavenumber filter is a "clean" choice for subgrid-scale dissipation. Horizontal eddy viscosity is denoted by $\nu$. The perturbation streamfunctions $\psi_{1}$ and $\psi_{2}$ satisfy

$q_{1}=\nabla^{2} \psi_{1}+\frac{\left(\psi_{2}-\psi_{1}\right)}{(1+\delta) L_{d}^{2}}, \quad q_{2}=\nabla^{2} \psi_{2}+\frac{\delta\left(\psi_{1}-\psi_{2}\right)}{(1+\delta) L_{d}^{2}}$,

where $\delta=H_{1} / H_{2}$, the ratio of upper to lower layer depths, and the first baroclinic mode deformation radius $L_{d}$ is defined as in Flierl (1978). The imposed mean PV gradients are

$$
\frac{\partial \overline{q_{1}}}{\partial y}=\frac{\left(\overline{u_{1}}-\overline{u_{2}}\right)}{(1+\delta) L_{d}^{2}}, \quad \frac{\partial \overline{q_{2}}}{\partial y}=\frac{\delta\left(\overline{u_{2}}-\overline{u_{1}}\right)}{(1+\delta) L_{d}^{2}} .
$$

The bottom Ekman drag coefficient $R_{2}$ is determined by the bottom boundary layer thickness $d_{\text {Ekman }}=\sqrt{A / f_{0}}$ via

$$
R_{2}=\frac{f_{0} d_{\text {Ekman }}}{2 H_{2}},
$$

where $f_{0}$ is the Coriolis parameter and $A$ is the vertical eddy viscosity (cf. Vallis 2006).

The barotropic (BT) and baroclinic (BC) streamfunctions are defined as in Flierl (1978):

$$
\psi_{\mathrm{BT}}=\frac{\delta \psi_{1}+\psi_{2}}{1+\delta}, \quad \psi_{\mathrm{BC}}=\frac{\sqrt{\delta}\left(\psi_{1}-\psi_{2}\right)}{1+\delta}
$$

The barotropic kinetic energy $\left(\mathrm{KE}_{\mathrm{BT}}\right)$ and baroclinic kinetic energy $\left(\mathrm{KE}_{\mathrm{BC}}\right)$ are given by

$$
\begin{aligned}
\mathrm{KE}_{\mathrm{BT}} & =\iint \frac{1}{2}\left|\nabla \psi_{\mathrm{BT}}\right|^{2} d x d y, \\
\mathrm{KE}_{\mathrm{BC}} & =\iint \frac{1}{2}\left|\nabla \psi_{\mathrm{BC}}\right|^{2} d x d y .
\end{aligned}
$$

The depth-averaged energy equation in physical space is obtained from multiplication of (2) by $-\delta \psi_{1} /(1+\delta)$, multiplication of (3) by $-\psi_{2} /(1+\delta)$, integration over the domain, and addition of the results, yielding:

$$
\begin{aligned}
\frac{\partial}{\partial t} \iint & \frac{1}{2}\left[\frac{\delta\left(\nabla \psi_{1}\right)^{2}}{1+\delta}+\frac{\left(\nabla \psi_{2}\right)^{2}}{1+\delta}+\frac{\delta\left(\psi_{1}-\psi_{2}\right)^{2}}{(1+\delta)^{2} L_{d}^{2}}\right] d x d y \\
= & \frac{\delta\left(\overline{u_{1}}-\overline{u_{2}}\right)}{(1+\delta)^{2} L_{d}^{2}} \iint \psi_{1} \frac{\partial \psi_{2}}{\partial x} d x d y-\frac{R_{2}}{1+\delta} \iint\left(\nabla \psi_{2}\right)^{2} d x d y \\
& -\nu \iint\left[\frac{\delta\left(\nabla^{2} \psi_{1}\right)^{2}}{1+\delta}+\frac{\left(\nabla^{2} \psi_{2}\right)^{2}}{1+\delta}\right] d x d y+w f
\end{aligned}
$$

where the first term on the right-hand side represents energy production, the second term represents energy dissipation by bottom friction, the third represents dissipation by eddy viscosity, and here $w f$ represents energy dissipation by the wavenumber filter.

Three nondimensional parameters control the behavior of the two-layer QG model. Following Flierl (1978) and Fu and Flierl (1980), the stratification parameter $\delta$ is set to a representative midlatitude oceanic value of 0.2 in all of the runs here. The nondimensional linear bottom friction strength is $F_{L}=R_{2} L_{d} /\left(\bar{u}_{1}-\bar{u}_{2}\right)$. In simulations performed with viscosity, the nondimensional viscosity is $\nu /\left[L_{d}\left(\bar{u}_{1}-\bar{u}_{2}\right)\right]$. For the sake of convenience we will use $\nu$ to denote both dimensional and nondimensional viscosity; the reader can discern which of the two is meant by context. 
(a) $\psi_{1}, v=0$

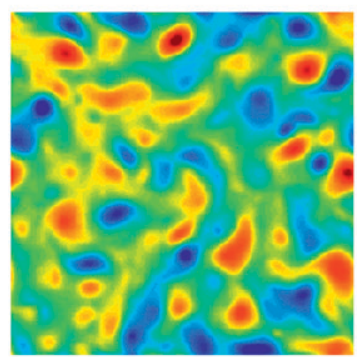

(c) $\psi_{1}, v=0.01$
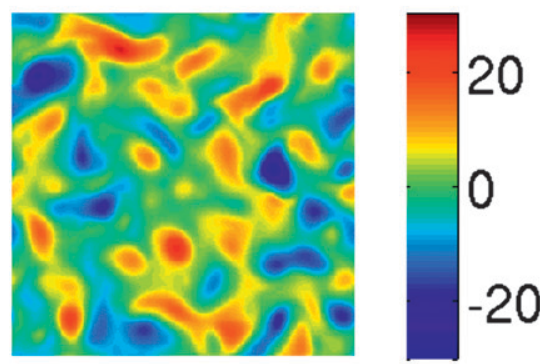

(e) $\psi_{1}, v=0.1$

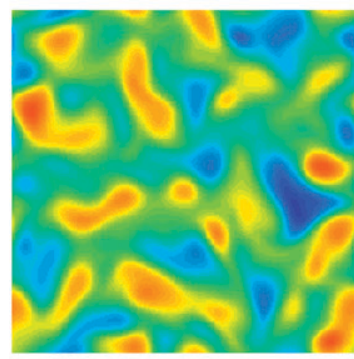

(g) $\psi_{1}, v=1$
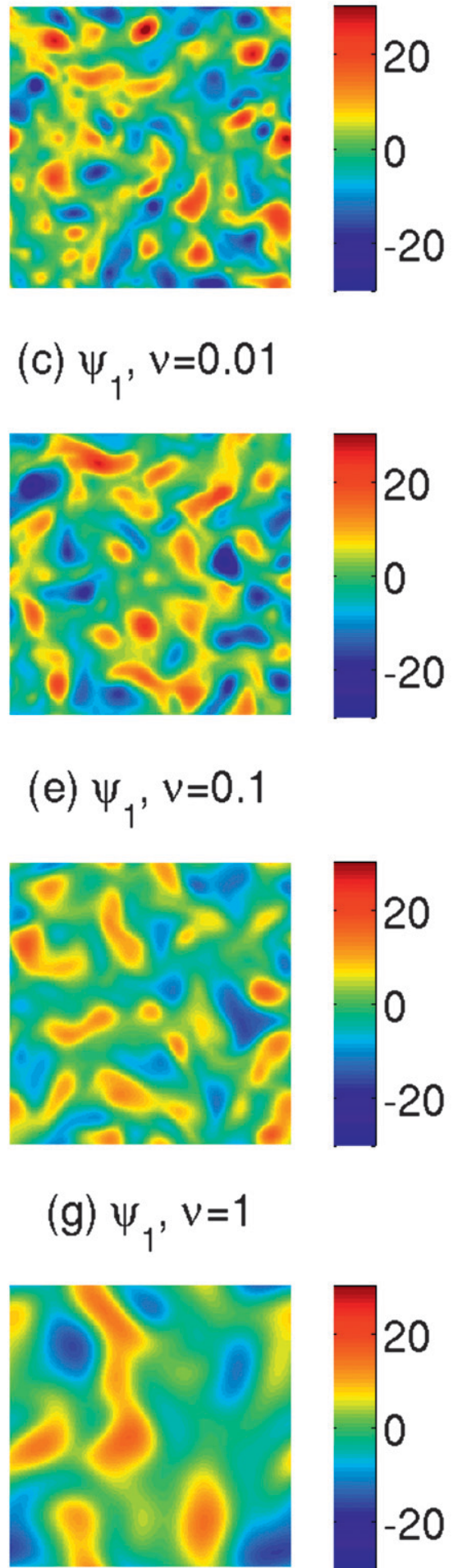

(b) $q_{1}, v=0$
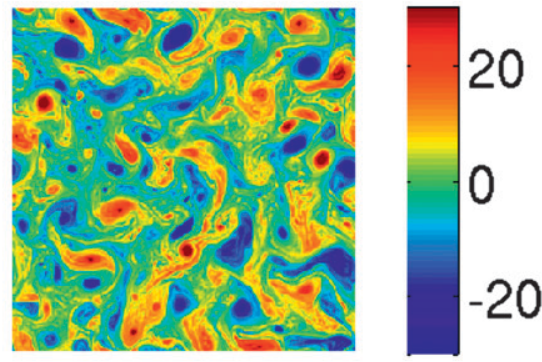

(d) $q_{1}, v=0.01$
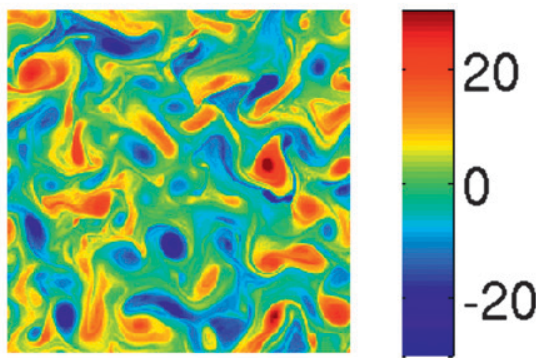

(f) $q_{1}, v=0.1$
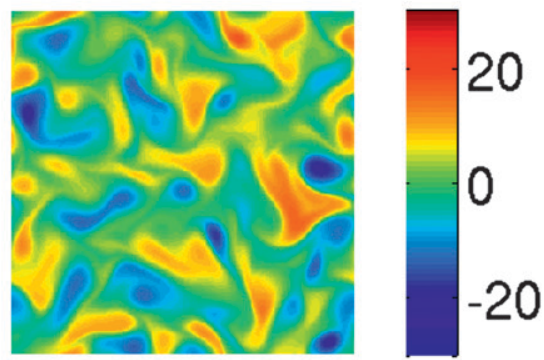

(h) $q_{1}, v=1$
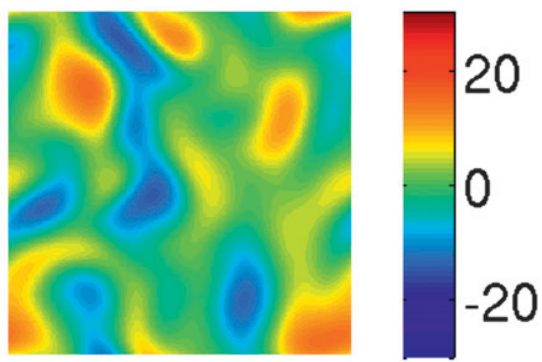

FIG. 2. Snapshots of upper-layer streamfunction $\psi_{1}$ and upper-layer potential vorticity $q_{1}$ for two-layer QG simulations with nondimensional bottom friction $F_{L}=0.4$ and nondimensional viscosity values $\nu$ of $0,0.01,0.1$, and 1 , respectively. The domain size is $20 \pi L_{d}$ on a side, where $L_{d}$ is the first baroclinic mode deformation radius. 
Figure 2 displays snapshots of the upper-layer streamfunction $\psi_{1}$ and upper layer potential vorticity $q_{1}$ in simulations with $F_{L}=0.4$ and nondimensional $\nu$ values of $0,0.01,0.1$, and 1 . The $\nu=0, F_{L}=0.4$ experiment is the "nominal" experiment of Arbic and Flierl (2004), that is, the experiment having amplitudes, horizontal scales, and vertical structure of eddy kinetic energy that agree reasonably well with observations in midlatitudes. The $\nu=0$, $F_{L}=0.4$ experiment consists of densely packed vortices (e.g., McWilliams 1984), in rough similarity to a typical snapshot of altimetrically measured SSH in the midocean (see, for instance, the model-altimeter comparison in Fig. 10 of Arbic and Flierl 2004). The vortices shown in Fig. 2 are not as dominant as the vortices in experiments with weaker bottom friction (see for instance Fig. 8 of Arbic and Flierl 2004). As is to be expected, Fig. 2 demonstrates that increasing horizontal eddy viscosity yields increasingly smooth streamfunction and potential vorticity fields.

\section{b. Spectral fluxes in idealized model}

As in Scott and Arbic (2007), which in turn followed Frisch (1995), we develop the spectral fluxes $\Pi(K)$ by letting

$$
\begin{aligned}
& \psi_{1}(x, y, t)=\sum_{k} \sum_{l} \widehat{\psi_{1}}(k, l, t) e^{i(k x+l y)}, \\
& \psi_{2}(x, y, t)=\sum_{k} \sum_{l} \widehat{\psi_{2}}(k, l, t) e^{i(k x+l y)},
\end{aligned}
$$

where $\hat{\psi}$ denotes a Fourier transform of $\psi$ in wavenumber space, and $k$ and $l$ are zonal and meridional wavenumbers, respectively. We neglect the subgridscale dissipation $w f$ for now since its impact on spectral fluxes is difficult to compute directly. We will compute its effects as a residual of the other terms in the spectral flux equations; later we will see that this residual is small. The Fourier transformed upper- and lower-layer governing equations are then

$$
\begin{aligned}
- & \frac{\partial}{\partial t}\left(K^{2}+\frac{1}{(1+\delta) L_{d}^{2}}\right) \widehat{\psi_{1}}(k, l, t)+\frac{\partial}{\partial t} \frac{1}{(1+\delta) L_{d}^{2}} \widehat{\psi_{2}}(k, l, t)+\overline{J\left(\psi_{1}, \nabla^{2} \psi_{1}\right)}(k, l, t)+\frac{1}{(1+\delta) L_{d}^{2}} \widehat{J\left(\psi_{1}, \psi_{2}\right)}(k, l, t) \\
& +\widehat{\Gamma_{1}}(k, l, t)=\nu K^{4} \widehat{\psi_{1}}(k, l, t), \\
- & \frac{\partial}{\partial t}\left(K^{2}+\frac{\delta}{(1+\delta) L_{d}^{2}}\right) \widehat{\psi_{2}}(k, l, t)+\frac{\partial}{\partial t} \frac{\delta}{(1+\delta) L_{d}^{2}} \widehat{\psi_{1}}(k, l, t)+\overline{J\left(\psi_{2}, \nabla^{2} \psi_{2}\right)}(k, l, t)+\frac{\delta}{(1+\delta) L_{d}^{2}} \widehat{J\left(\psi_{2}, \psi_{1}\right)}(k, l, t) \\
& +\widehat{\Gamma_{2}}(k, l, t)=R_{2} K^{2} \widehat{\psi_{2}}(k, l, t)+\nu K^{4} \widehat{\psi_{2}}(k, l, t),
\end{aligned}
$$

respectively, where $K^{2}=k^{2}+l^{2}$ and we have dropped the summation signs for simplicity.

We now multiply the Fourier transformed equations (13) and (14) by $-\delta \widehat{\psi}_{1}^{*}(k, l, t) /(1+\delta)$ and $-\widehat{\psi_{2}^{*}}(k, l, t) /(1+\delta)$, respectively, where the superscript asterisk represents a complex conjugate, add the two results together, take the real part, assume a statistically steady state, and take averages over time (so that the $\partial / \partial t$ terms drop out), to obtain the depth-averaged spectral energy equation

$$
\begin{aligned}
& T_{\mathrm{KE}, 1}(k, l)+T_{\mathrm{KE}, 2}(k, l)+T_{\mathrm{APE}}(k, l)+T_{\text {forcing }}(k, l) \\
& \quad+T_{\text {friction }}(k, l)+T_{\text {viscosity }}(k, l)=0
\end{aligned}
$$

In (15) the spectral transfers of upper and lower layer kinetic energy are

$$
T_{\mathrm{KE}, 1}(k, l)=\operatorname{Re}\left\langle\frac{\delta}{1+\delta} \widehat{\psi_{1}^{*}}(k, l, t) \widehat{J\left(\psi_{1}, \nabla^{2} \psi_{1}\right)}(k, l, t)\right\rangle,
$$

and

$$
\begin{aligned}
T_{\mathrm{KE}, 2}(k, l)= & \operatorname{Re}\left\langle\frac{1}{1+\delta} \widehat{\psi_{2}^{*}}(k, l, t)\right. \\
& \left.\times \overline{J\left(\psi_{2}, \nabla^{2} \psi_{2}\right)}(k, l, t)\right\rangle,
\end{aligned}
$$

respectively, where Re denotes the real part of a complex number, and the bracket operator \langle\rangle represents a time average. The spectral transfer of available potential energy is

$$
\begin{aligned}
T_{\mathrm{APE}}(k, l)= & \operatorname{Re}\left\langle\frac{\delta}{(1+\delta)^{2} L_{d}^{2}} \overline{\left(\psi_{1}-\psi_{2}\right.}\right)^{*}(k, l, t) \\
& \left.\times \overline{J\left(\psi_{1}, \psi_{2}\right)}(k, l, t)\right\rangle .
\end{aligned}
$$

The spectral transfer due to the imposed mean flow forcing is

$$
\begin{aligned}
T_{\text {forcing }}(k, l)= & \operatorname{Re}\left\langle\frac{\delta}{1+\delta} \widehat{\psi_{1}^{*}}(k, l, t) \widehat{\Gamma_{1}}(k, l, t)\right\rangle \\
& +\operatorname{Re}\left\langle\frac{1}{1+\delta} \widehat{\psi_{2}^{*}}(k, l, t) \widehat{\Gamma_{2}}(k, l, t)\right\rangle,
\end{aligned}
$$


the spectral transfer due to bottom Ekman friction is

$$
T_{\text {friction }}(k, l)=-\frac{1}{1+\delta} R_{2} K^{2}\left\langle\widehat{\psi_{2}^{*}}(k, l, t) \widehat{\psi_{2}}(k, l, t)\right\rangle
$$

and the spectral transfer due to eddy viscosity is

$$
\begin{aligned}
T_{\text {viscosity }}(k, l)= & -\frac{\delta}{1+\delta} \nu K^{4}\left\langle\widehat{\psi_{1}^{*}}(k, l, t) \widehat{\psi_{1}}(k, l, t)\right\rangle \\
& -\frac{1}{1+\delta} \nu K^{4}\left\langle\widehat{\psi_{2}^{*}}(k, l, t) \widehat{\psi_{2}}(k, l, t)\right\rangle .
\end{aligned}
$$

As noted previously, the effects of the wavenumber filter on the energy budgets will be calculated as a residual. Because of the filter, in practice, the right-hand side of (15) is not identically zero.

Spectral fluxes are defined as semi-infinite integrals of the spectral transfers. For example

$$
\Pi_{\mathrm{KE}, 1}(K)=\iint_{k^{2}+l^{2} \geq K^{2}} T_{\mathrm{KE}, 1}(k, l) d k d l,
$$

and more generally,

$$
\Pi_{\text {term }}(K)=\iint_{k^{2}+l^{2} \geq K^{2}} T_{\text {term }}(k, l) d k d l,
$$

where the subscript "term" could denote "KE, 1," "KE, 2," "APE," "forcing," "friction," or "viscosity." Following the terminology of Scott and Arbic (2007), we will call the budget obtained by summing the spectral fluxes $\Pi(K)$ [as in Eq. (15), but with every " $T$ " replaced by a " $\Pi$ "] the "integral energy budget." Note that since we are using the depth-averaged energy equation there are no spectral transfers or fluxes representing energy transfer between layers.

\section{c. Impact of viscosity on idealized model}

Our first look at the spectral fluxes $\Pi_{\mathrm{KE}, 1}(K)$ of upperlayer kinetic energy in the QG model is displayed in Fig. 3. Fluxes are shown for $F_{L}=0.4$ simulations with four different nondimensional $\nu$ values $(0,0.01,0.1$, and 1$)$. Figure 3 a presents the fluxes normalized by $\left(\bar{u}_{1}-\bar{u}_{2}\right)^{3} / L_{d}$, while in Fig. $3 b$ all spectral flux curves are normalized by their minimum (negative) $\Pi(K)$ value at larger scales, so that the minimum values in the normalized curves are always -1 . The spectral flux $\Pi_{\mathrm{KE}, 1}(K)$ from the simulation with $F_{L}=0$ and zero viscosity-with only a wavenumber filter present to absorb the enstrophy cascade-is represented by black curves in Figs. $3 \mathrm{a}$ and $3 \mathrm{~b}$. There is a vigorous inverse cascade at scales near $L_{d}$-both scales slightly larger than $L_{d}$ and scales slightly smaller than $L_{d}$-but very little forward cascade at small scales. The small forward cascade at small scales is consistent with the (a)

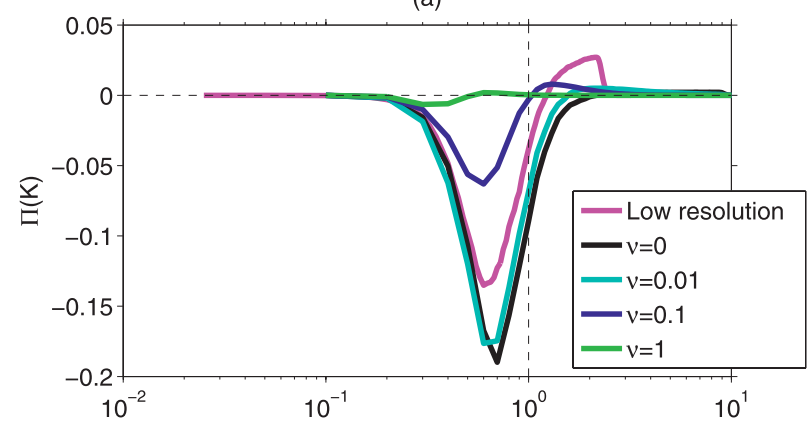

(b)

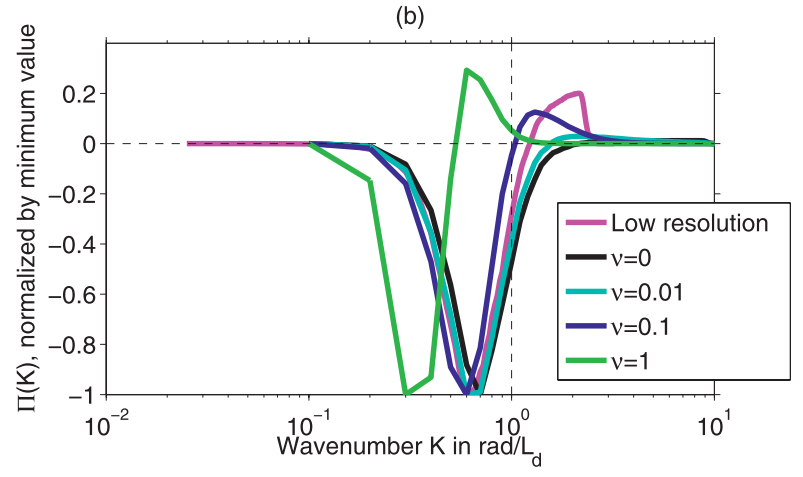

FIG. 3. Spectral kinetic energy flux $\Pi_{\mathrm{KE}, 1}(K)$ in upper layer of two-layer QG model, for low-resolution simulation with zero viscosity, and simulations with varying nondimensional viscosity $\nu$. The low-resolution experiment was run in a larger domain with the same number of gridpoints as in the other experiments, such that scales near $L_{d}$ were not as well resolved. In all simulations shown above the nondimensional bottom friction $F_{L}$ is 0.4 . In (a), the fluxes are normalized by $\left(\bar{u}_{1}-\bar{u}_{2}\right)^{3} / L_{d}$. In (b), all flux curves are normalized so that the minimum value in the negative lobe is -1 .

fact that almost all of the energy dissipation is accomplished by bottom drag in the $\nu=0$ simulation, and very little is accomplished by the wavenumber filter.

In the $\nu=0.01$ experiment (cyan curves) there is a small forward cascade. In the experiments with nondimensional $\nu$ values of 0.1 and 1 (blue and green curves, respectively), there is a substantial forward cascade (positive lobe) at smaller scales, in qualitative agreement with the shape seen in the observations (Fig. 1). ${ }^{2}$ At first glance this suggests that the forward cascades seen in altimetry data could be robust and due to processes that can roughly be characterized with a horizontal eddy viscosity. We therefore ask whether 0.1 is a plausible value for the nondimensional $\nu$ in the midocean. If we take the dimensional horizontal eddy viscosity to equal $50 \mathrm{~m}^{2} \mathrm{~s}^{-1}$, as argued by Polzin (2008,

\footnotetext{
${ }^{2}$ Note that the forward cascade is present in both the barotropic and baroclinic modes (not shown).
} 
2010), together with typical midlatitude open-ocean gyre values of $L_{d}=50 \mathrm{~km}$ (Richman et al. 1977) and $\bar{u}_{1}-\bar{u}_{2}=1 \mathrm{~cm} \mathrm{~s}^{-1}$ (Stammer 1997), we obtain a nondimensional $\nu$ of 0.1 , suggesting that this may indeed be a plausible value. (Note, however, that the nondimensional $\nu$ values inferred later from the realistic model are somewhat smaller.) The spectral fluxes computed from experiments with larger nondimensional $\nu$ values display a shift of the zero crossings to lower wavenumbers (seen more easily in Fig. 3b). In the $\nu=0.1$ experiment the zero crossing lies very close to the deformation wavenumber, as in the observational $\Pi(K)$ curve shown in Fig. 1 . In the $\nu=0$ experiment there is some negative flux (inverse cascade) occurring for wavenumbers higher than the deformation wavenumber.

The magenta curves in Figs. $3 \mathrm{a}$ and $3 \mathrm{~b}$ denote $\Pi_{\mathrm{KE}, 1}(K)$ computed from a $\nu=0, F_{L}=0.4$ simulation in which the number of gridpoints is the same $-256^{2}$-but in which the domain is 4 times larger on each side. The horizontal grid resolution of this run is therefore lower. In the low-resolution run the spectral fluxes point to a forward cascade of energy at small scales toward yet smaller scales, and the subgrid-scale dissipation accomplishes a substantial fraction (about 14\%) of the energy dissipation. However, since this cascade is not present in the higher-resolution $\nu=0$ simulation, the forward flux is merely a result of insufficent horizontal resolution. Note that lower horizontal resolution is typical of many QG turbulence studies, for example, Larichev and Held (1995), since they often focus on the inverse cascade and utilize large domains to give the inverse cascade room to proceed. We will return to this theme, of the effects of insufficient horizontal resolution, shortly. In the remainder of the discussion in this section we return to experiments performed at higher horizontal resolution, though in some analyses the model results are filtered after the run is completed.

Horizontal eddy viscosity significantly alters the QG model energy budget. This can be seen in Fig. 4, which displays the depth-integrated integral energy budget in the simulation without viscosity (Fig. 4a; black curves in Fig. 3) and in the simulation with parameters otherwise equal, but with a nondimensional $\nu$ value of 0.1 (Fig. 4b; blue curves in Fig. 3). In the simulation without viscosity, bottom drag accomplishes nearly all of the energy dissipation. In the simulations with $\nu=0.1$, about half of the energy dissipation is accomplished by viscosity. This is in reasonable agreement with a regional mesoscale energy budget derived from observations (Polzin 2010), which suggests that eddy viscosity and bottom drag dissipate similar amounts of energy. Note that in Fig. 4 the upper- and lower-layer contributions to the forcing,

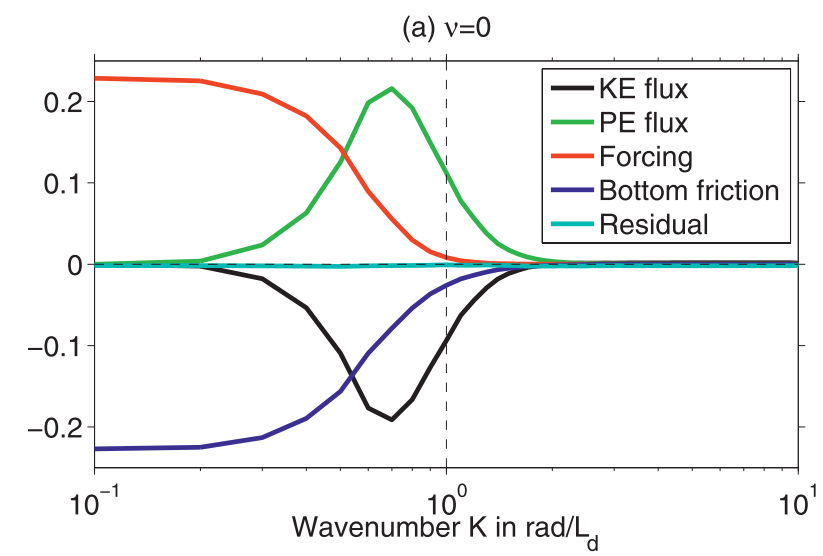

(b) $v=0.1$

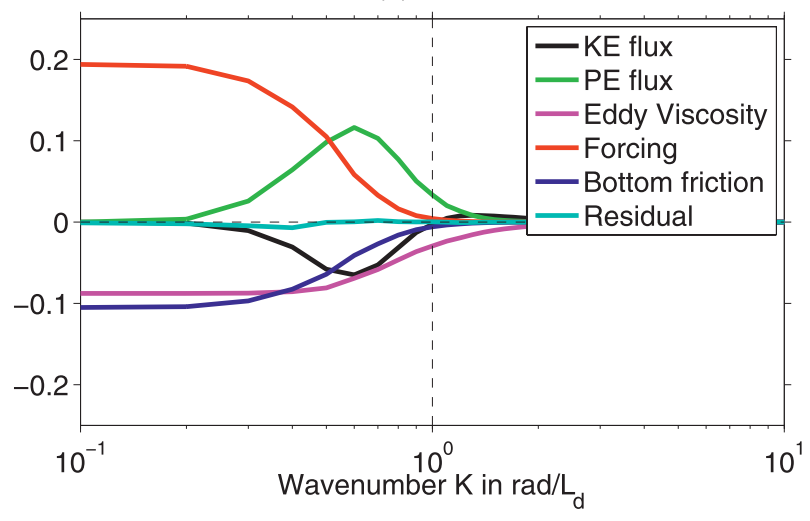

FIG. 4. Integral energy budgets-budgets of spectral fluxes $\Pi(K)$ - of two-layer QG simulations with $F_{L}=0.4$ and nondimensional horizontal eddy viscosity values $\nu$ of (a) 0 and (b) 0.1 . In both subplots the cyan line denotes the residual of the other terms, assumed dominated by the wavenumber filter. All terms normalized by $\left(\bar{u}_{1}-\bar{u}_{2}\right)^{3} / L_{d}$.

viscosity, and kinetic energy fluxes have been added together; the upper layer dominates these sums.

Next, we briefly discuss the sensitivity of QG turbulence to viscosity. Figure 5 displays various eddy statistics as a function of nondimensional $\nu$. We use three different values of $F_{L}(0.2,0.4$, and 0.8$)$. In the first five subplots of Fig. 5 extra horizontal lines denote rough ranges of values observed at different locations in the ocean (see also Table 1; note that the range of observed FtoI values-i.e. the ratio of peak forward to peak inverse fluxes-is taken from the regional AVISO calculations to be described in section 4). Figures 5a-d display relatively flat sensitivity to $\nu$ until $\nu$ takes on values of order one and larger. The large $\nu$ regime is not likely to be relevant to the ocean but is included for the sake of completeness in the sensitivity study. For $\nu$ of order one and smaller, the surface eddy kinetic energy behaves as one might expect-it generally decreases with increasing $\nu$ and with increasing $F_{L}$ (Fig. 5a). The length scale $L_{1}$ of upper layer kinetic energy, defined by 
(a) Surface eddy KE

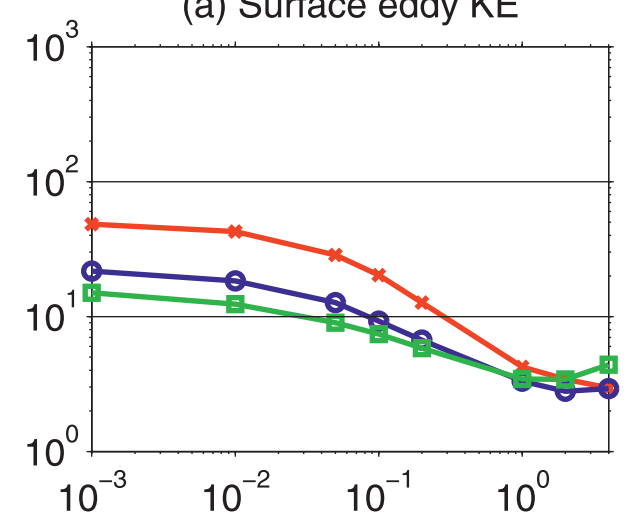

(c) $\mathrm{KE}_{\mathrm{BC}} / \mathrm{KE}_{\mathrm{BT}}$

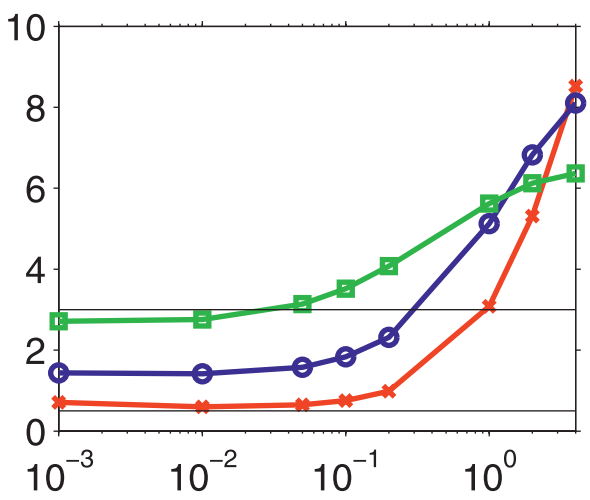

(e) Peak forward to inverse flux

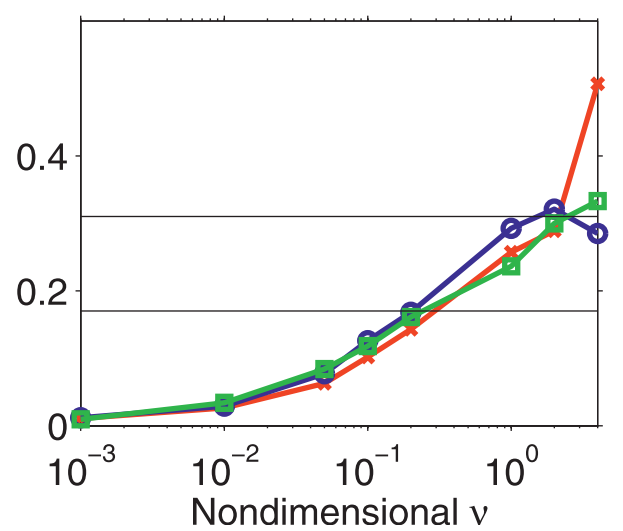

(b) $L_{1} / L_{d}$

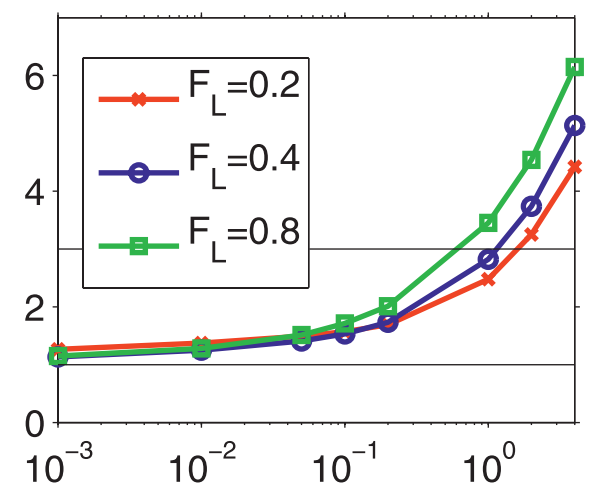

(d) $\left\langle u_{1}^{2}+v_{1}^{2}>l<u_{2}^{2}+v_{2}^{2}>\right.$

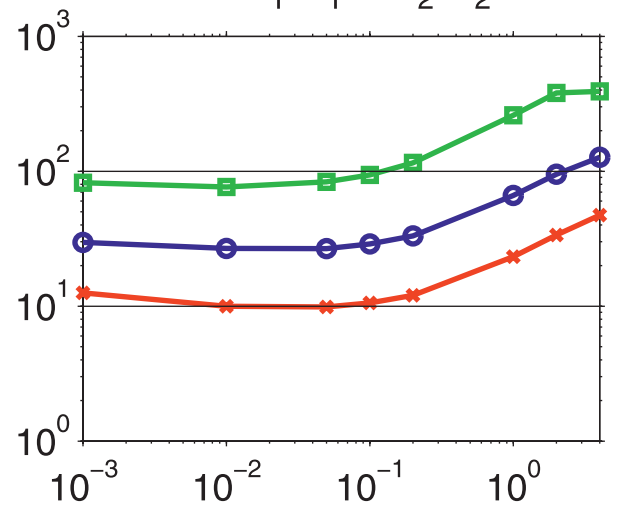

(f) Energy dissipation by viscosity/production

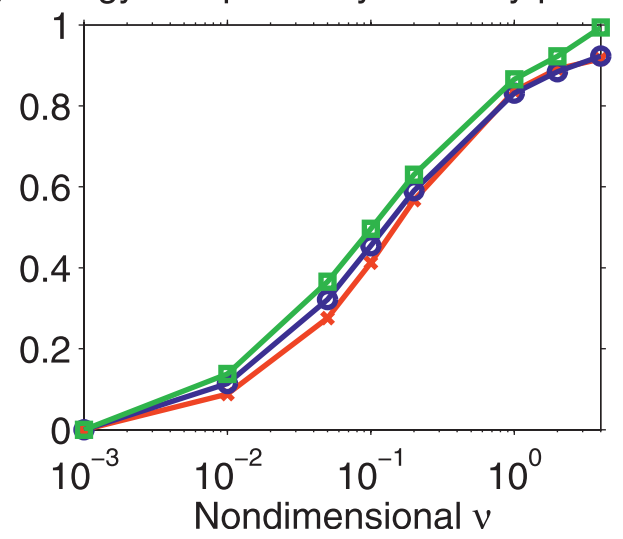

FIG. 5. Various time-averaged eddy statistics in two-layer QG turbulence model, vs nondimensional $\nu$ ( $x$ axes). The $x$ axes are $\log$ axes; experiments with $\nu=0$ are assigned to $\nu=10^{-3}$ so that they can be placed onto these log axes. Three different nondimensional bottom friction $\left(F_{L}\right)$ values are used; see legend in subplot $\mathrm{b}$. Extra horizontal lines denote rough ranges of values inferred from observations (see text and Table 1). (a) Surface eddy kinetic energy (KE) $0.5\left\langle u_{1}^{2}+v_{1}^{2}\right\rangle$, normalized by mean flow kinetic energy $0.5\left(\bar{u}_{1}-\bar{u}_{2}\right)^{2}$. (b) Ratio of upper layer length scale $L_{1}$ (see text) to $L_{d}$. (c) Ratio $\mathrm{KE}_{\mathrm{BC}} / \mathrm{KE}_{\mathrm{BT}}$ of baroclinic to barotropic kinetic energy. (d) Ratio of time-averaged squared upper-layer velocities $\left\langle u_{1}^{2}+v_{1}^{2}\right\rangle$ to time-averaged squared lower layer velocities $\left\langle u_{2}^{2}+v_{2}^{2}\right\rangle$. (e) Ratio FtoI of peak forward to inverse flux [see Eq. (1)]. (f) Ratio $E_{\nu}$ of energy dissipation by viscosity to total energy production. 
TABLE 1. Various time-averaged eddy statistics in two-layer QG turbulence model, in selected runs with different values of $\nu$ and $F_{L}$ as given. The $\nu=0, F_{L}=0.4$ simulation is the "nominal solution" in Arbic and Flierl (2004), which they argued compared well to ocean observations. See text and caption to Fig. 5 for definitions of symbols used in this Table. Rough ranges of observed values are also given. Sources for the ranges of observed values are given by superscripts below.

\begin{tabular}{lccccc}
\hline \hline & Surface KE & $\frac{L_{1}}{L_{d}}$ & $\frac{\mathrm{KE}_{\mathrm{BC}}}{\mathrm{KE}_{\mathrm{BT}}}$ & $\frac{\left\langle u_{1}^{2}+v_{1}^{2}\right\rangle}{\left\langle u_{2}^{2}+v_{2}^{2}\right\rangle}$ & FtoI \\
\hline Observations & $10-100^{\mathrm{a}}$ & $1-3^{\mathrm{b}}$ & $0.5-3^{\mathrm{c}}$ & $10-100^{\mathrm{d}}$ & $0.17-0.31^{\mathrm{e}}$ \\
$\nu=0, F_{L}=0.4$ & 21 & 1.1 & 1.5 & 31 & 0.012 \\
$\nu=0.05, F_{L}=0.4$ & 13 & 1.4 & 1.6 & 27 & 0.077 \\
$\nu=0.1, F_{L}=0.4$ & 9.3 & 1.5 & 1.8 & 29 & 0.13 \\
$\nu=0.05, F_{L}=0.2$ & 29 & 1.5 & 0.65 & 9.9 & 0.32 \\
$\nu=0.1, F_{L}=0.2$ & 20 & 1.6 & 0.75 & 11 & 0.063 \\
$\nu=0.2, F_{L}=0.2$ & 13 & 1.7 & 0.98 & 0.12 & 0.10 \\
$\nu=0.01, F_{L}=0.8$ & 12 & 1.3 & 2.8 & 77 & 0.41 \\
\hline
\end{tabular}

${ }^{\mathrm{a}}$ Wunsch (2001).

b Stammer (1997); see also Fig. 9b of Arbic et al. (2007) for observed eddy length scales estimated using the same method used here to compute length scales from the QG models.

${ }^{\mathrm{c}}$ Wunsch (1997).

d Schmitz (1996).

e Calculations in section 4.

$$
L_{1}=\left[\frac{\iint \sqrt{k^{2}+l^{2}} E(k, l) d k d l}{\iint E(k, l) d k d l}\right]^{-1},
$$

where $\sqrt{k^{2}+l^{2}}=K$ is isotropic wavenumber, and $E(k, l)$ is the wavenumber spectrum of upper layer kinetic energy, is plotted in Fig. 5b. Increasing nondimensional $\nu$ leads to increasing eddy length scales, in keeping with the smoothing effect of viscosity seen in Fig. 2. For large $\nu$ values the flow becomes increasingly baroclinic (Fig. 5c) and increasingly surface intensified (Fig. 5d). The degree of surface intensification increases as the bottom friction $F_{L}$ increases, and is also significantly impacted by the value of $\delta$ (Arbic and Flierl 2004). As nondimensional values of $\nu$ increase, the FtoI values generally increase (Fig. 5e). Accordingly, the ratio $E_{\nu}$ of energy dissipated by viscosity to total energy production-where both are taken from Eq. (11)-increases steadily with increasing viscosity $\nu$, approaching unity for nondimensional $\nu$ values of order one or larger. Figures $5 \mathrm{e}$ and $5 \mathrm{f}$ demonstrate that even $\nu$ values as small as 0.01 yield a nonnegligible forward kinetic energy cascade and viscous dissipation.

As seen in Figs. 5a-d, the amplitude, horizontal scale, and vertical structure of the eddy kinetic energy in the QG model generally lie outside of observed ranges for nondimensional $\nu$ values larger than order one, and inside or at least near the observed range for $\nu$ values of order one and smaller. As seen in Fig. 5e, the FtoI values lie near the range seen in present-generation altimeter data only for $\nu$ values of about 0.1 or larger. However, the observed range in Fig. 5e may be too large for reasons to be discussed shortly. Lowering the observed FtoI values would imply that the small $\nu$ QG experiments match the FtoI values in the data more closely.

To complement Fig. 5, Table 1 displays values of the eddy statistics for selected QG experiments, in which the statistics in Figs. 5a-d lie close to, or inside, the ranges of observed values. Taken together, Fig. 5 and Table 1 indicate that it is possible for the amplitudes, horizontal scales, and vertical structure of eddies in QG turbulence simulations to match values in observations reasonably well, while still having a nonnegligible forward cascade and viscous dissipation.

\section{d. Effect of filtering on spectral fluxes in idealized model}

Next we investigate the effect of filtering the $\psi_{1}$ fields on the computations of $\Pi_{\mathrm{KE}, 1}(K)$ from idealized $\mathrm{QG}$ model output. We are particularly interested in simulations that do not display a forward cascade in the unfiltered case, as we wish to discern whether filtering will yield an artificial forward cascade. Note that filtering is performed on the model output after the model has been run, not during the run itself. Figure 6 shows $\Pi_{\mathrm{KE}, 1}(K)$ computed from the $\nu=0, F_{L}=0.4$ QG simulation (black curves in Fig. 3), and from filtered versions of this simulation. The spatial filter goes to zero 6 grid points from the central grid point, meaning it has a "full-width half maximum" (width at the half-power point) of 3.4 grid points, or a wavelength of $0.83 L_{d}$. The time filter is a Blackman filter which goes to zero 4 units of nondimensional time $L_{d} /\left(\bar{u}_{1}-\bar{u}_{2}\right)$ from the central time. Filtering in space, or in time, diminishes the negative lobe of the spectral flux (inverse cascade) substantially. Filtering in both space and time further diminishes the negative lobe. Widening the time filter to 9 units of 


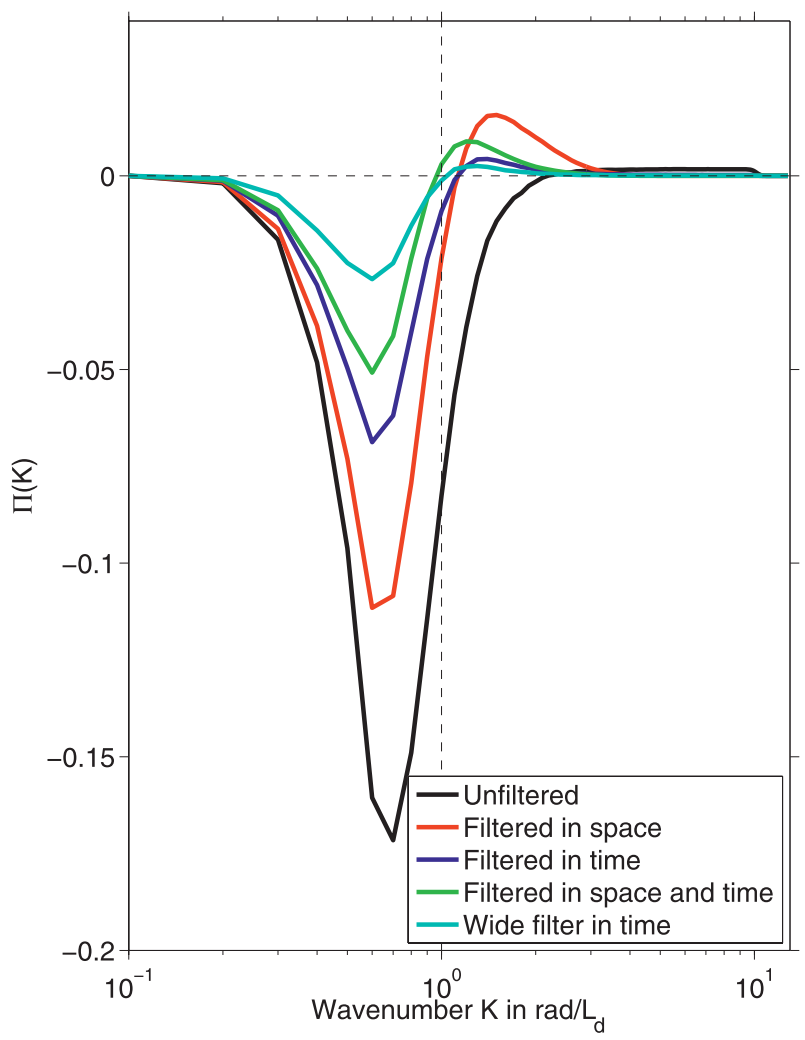

FIG. 6. Spectral flux $\Pi_{\mathrm{KE}, 1}(K)$ of upper-layer kinetic energy computed from the $\nu=0, F_{L}=0.4$ two-layer QG simulation ("unfiltered") and from filtered versions of this simulation. See text for descriptions of filters used. All fluxes normalized by $\left(\bar{u}_{1}-\bar{u}_{2}\right)^{3} / L_{d}$.

nondimensional time diminishes the inverse cascade even more. Most importantly, we see that a simulation with $\nu=0$ can see a substantial forward flux at smaller scales if the $\psi_{1}$ field is first spatially and/or temporally filtered before $\Pi_{\mathrm{KE}, 1}(K)$ is computed, whereas little forward flux is seen in the fluxes computed from unfiltered $\nu=0$ model output. This suggests that spectral fluxes computed from the present-day AVISO gridded altimeter product, which is highly filtered, could display a forward cascade as an artifact of insufficient horizontal resolution. In contrast, the existence of the inverse cascade is robust to the filtering exercise, though the magnitude of the inverse cascade is not. Finally, we note that filtering, like an increase of viscosity, tends to shift the zero crossings of the $\Pi_{\mathrm{KE}, 1}(K)$ curves toward lower wavenumbers.

\section{Results from the realistic model and gridded satellite altimeter data}

\section{a. Description of realistic model}

We use three full years of output (2001-03) from the forward (nonassimilative) version of the Naval Research
Laboratory (NRL) Layered Ocean Model (NLOM). Data-assimilative versions of NLOM are in current use as a U.S. Navy operational model. Shriver et al. (2007) and references therein describe the NLOM grid, wind forcing, and other model details. The model horizontal resolution is $1 / 32^{\circ}$ in latitude and $45 / 1024^{\circ}$ in longitude, on a model grid extending from $72^{\circ} \mathrm{S}$ to $65^{\circ} \mathrm{N}$. For simplicity, we refer to the simulations as having $1 / 32^{\circ}$ resolution. NLOM is based on the primitive equation model of Hurlburt and Thompson (1980), but with greatly expanded capability (Wallcraft et al. 2003). It has 6 dynamical layers and a bulk mixed layer.

Strengths of NLOM for the current study include the simplicity of the subgrid-scale closures it employs relative to the closures employed in other widely used realistic models. In the NLOM momentum equations, the only subgrid scale dissipation utilized is a horizontal eddy viscosity of $20 \mathrm{~m}^{2} \mathrm{~s}^{-1}$, comparable to the values inferred from observations by Polzin (2010). A horizontal eddy diffusivity of equal strength, acting on density, is also employed in the model. In addition, horizontal biharmonic diffusivities of $2.5 \times 10^{7} \mathrm{~m}^{4} \mathrm{~s}^{-1}$ are employed on density and layer thickness. The only vertical (between layer) process available in NLOM is interfacial friction, which is not turned on in the simulations examined for this paper.

\section{b. Satellite altimeter data}

We use 840 snapshots of the two-satellite AVISO $13^{\circ}$ Mercator grid "reference" product (Le Traon et al. 1998; Ducet et al. 2000), beginning with 14 October 1992 and ending with 12 November 2008. The AVISO record we utilize is much longer than the NLOM record (16 versus 3 years) but has a much lower temporal sampling rate ( 7 days versus $6 \mathrm{~h}$ ). The spatial resolution is also much lower $\left(1 / 3^{\circ}\right.$ versus $\left.1 / 32^{\circ}\right)$.

\section{c. Regions used in analyses}

For both the realistic model and satellite altimeter analyses, we compute spectral fluxes over six regions. The regions are shown in Fig. 7, against backdrops of SSH and SSH anomaly snapshots from the model and altimeter data, respectively. Two of the regions-"midlatitude southeast Pacific" and "high latitude southeast Pacific" are relatively quiescent. Four of the regions-"Agulhas," off the tip of southern Africa, "Malvinas," in the western South Atlantic, "Gulf Stream," in the western North Atlantic, and "Kuroshio," in the western North Pacificcontain strong western boundary currents and mesoscale eddy activity, as seen in Fig. 7. The correspondence between regions in the AVISO versus NLOM analysis is not exact, because of the lack of grid points in shallow waters in NLOM (see Shriver et al. 2007 and references therein). 

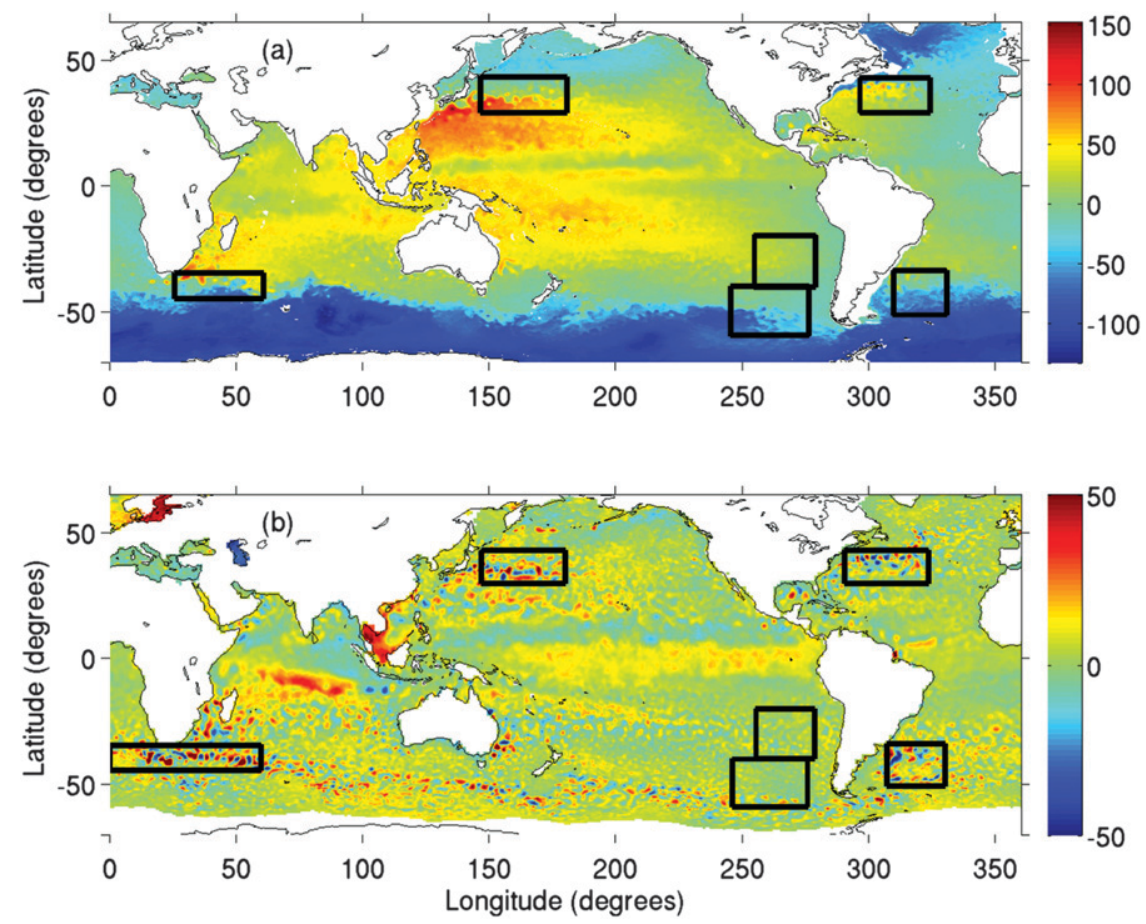

FIG. 7. Regions used to compute spectral fluxes $\Pi_{\mathrm{KE}, 1}(K)$ of surface ocean geostrophic kinetic energy in (a) $1 / 32^{\circ}$ NLOM output and (b) $1 / 3^{\circ}$ Mercator AVISO gridded satellite altimeter data. The NLOM regions are highlighted against the 15 Aug 2002 snapshot of sea surface height $(\mathrm{cm})$ in the model, while the AVISO regions are highlighted against the 20 Dec 2006 snapshot of sea surface height anomaly $(\mathrm{cm})$ in AVISO. For the sake of comparison, for this figure the AVISO data was interpolated onto a $1 / 3^{\circ}$ regular latitude-longitude grid having the same latitudinal range as the NLOM grid.

The misfit between the boundaries of the NLOM versus AVISO regions is largest in the Agulhas.

\section{d. Spectral fluxes in realistic model and gridded altimeter data}

We now describe the spectral flux calculations performed with output from the realistic model (NLOM) and from gridded satellite altimeter data (AVISO). The NLOM sea surface heights are first low-pass filtered in time to remove motions with periods of 3 days or less. This is done because high-frequency motions cannot be geostrophic. For both the NLOM and AVISO analyses, we compute $\psi_{1}=g \eta / f$, where $\eta$ is the perturbation $\mathrm{SSH}$, $g=9.8 \mathrm{~m} \mathrm{~s}^{-2}$ is gravitational acceleration, and $f$ is the Coriolis parameter, which varies as the sine of the gridpoint latitude (Vallis 2006). Then for each of the six regions shown in Fig. 7, each snapshot of $\psi_{1}$ is detrended in space, with a two-dimensional least squares fit. Following this, the $\psi_{1}$ field is tapered in space with a twodimensional function constructed of nine overlapping Hanning windows in each spatial direction, where each window has a width one-fifth of the domain extent in that direction. This type of detrending and tapering is standard in computations of Fourier transforms of data in nonperiodic domains (Priestley 1981). The sensitivity to tapering will be discussed shortly. Fourier transforms are repeatedly utilized to compute derivatives and hence the term $J\left(\psi_{1}, \nabla^{2} \psi_{1}\right)$. For simplicity we base the increment lengths of the wavenumbers in the east-west direction on the length of the increments at the central latitude of the box. In other words, we do not account for the convergence of meridians in our spectral calculations. As in the $\mathrm{QG}$ calculations, the transfer

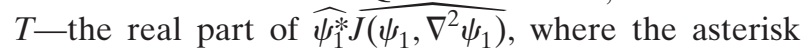
indicates the complex conjugate-is computed for all snapshots, and then averaged over the snapshots. An integration over the resulting time-averaged $T$ values for all wavenumbers greater than a given wavenumber $K$ yields the spectral flux $\Pi_{\mathrm{KE}, 1}(K)$ for that wavenumber.

Figures 8-10 display the spectral flux of surface ocean geostrophic kinetic energy $\Pi_{\mathrm{KE}, 1}(K)$ computed from the NLOM simulation, alongside fluxes computed from AVISO gridded altimeter data, for the six regions of interest. Note that in these more realistic computations $\Pi_{\mathrm{KE}, 1}(K)$ does not include the factor of $\delta /(1+\delta)$ utilized in section 3. This factor is dropped here for 
(a) Midlatitude SE Pacific

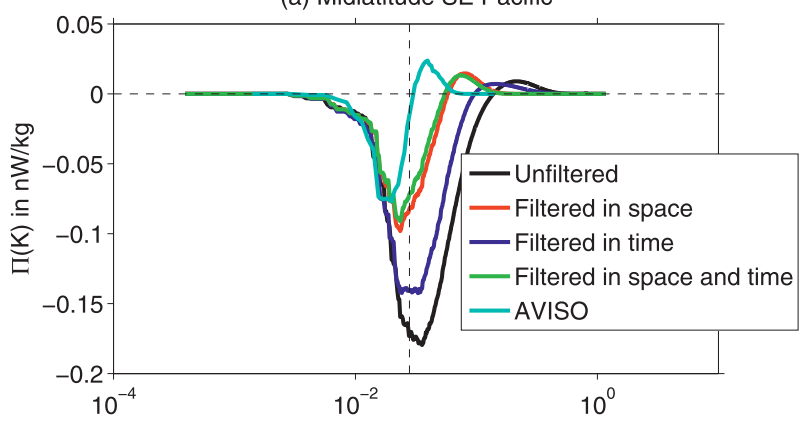

(b) High latitude SE Pacific

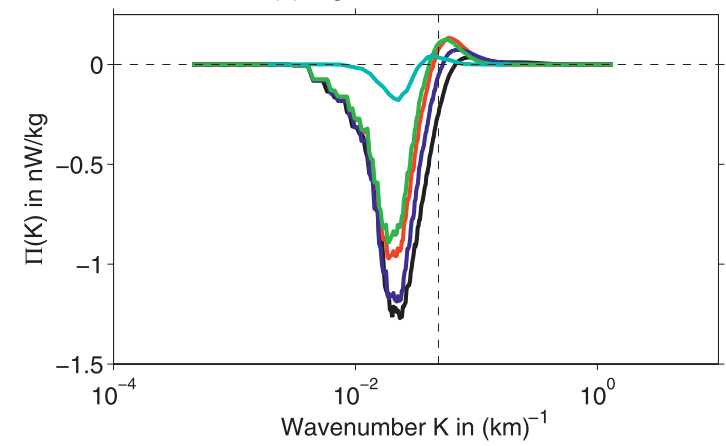

FIG. 8. Spectral flux $\Pi_{\mathrm{KE}, 1}(K)$ of surface ocean geostrophic kinetic energy in (a) midlatitude southeast Pacific and (b) highlatitude southeast Pacific, computed from AVISO (cyan curves) and from four versions of realistic NLOM simulation output: unfiltered (black curves), filtered in space (red curves), filtered in time (blue curves), and filtered in space and time (green curves). See text for details. Vertical dashed lines denote deformation wavenumber $1 / L_{d}$, where the deformation radius $L_{d}$ is taken from Chelton et al. (1998).

simplicity, since oceanic stratification varies from one location to another.

The black curves in Figs. 8-10 show the "unfiltered" NLOM fluxes-that is, the fluxes computed from NLOM output that is unadalterated except for the manipulations described in the first paragraph of this subsection. Consistent with the results of Schlösser and Eden (2007) and Tulloch et al. (2011), the spectral flux computed from a realistic high-resolution model has a prominent negative lobe, indicating an inverse cascade, at larger scales. The deformation wavenumbers $1 / L_{d}$, where $L_{d}$ is the first baroclinic mode deformation radius, are shown as vertical dashed lines on Figs. 8-10. The $L_{d}$ values are taken from the atlas of Chelton et al. (1998). For each of the six regions, we choose the $L_{d}$ value at the atlas gridpoint lying closest to the center of the AVISO region in question. In all six regions, NLOM displays a significant inverse cascade (negative spectral fluxes) over a band of wavenumbers higher than the deformation wavenumber, consistent with the behavior seen in

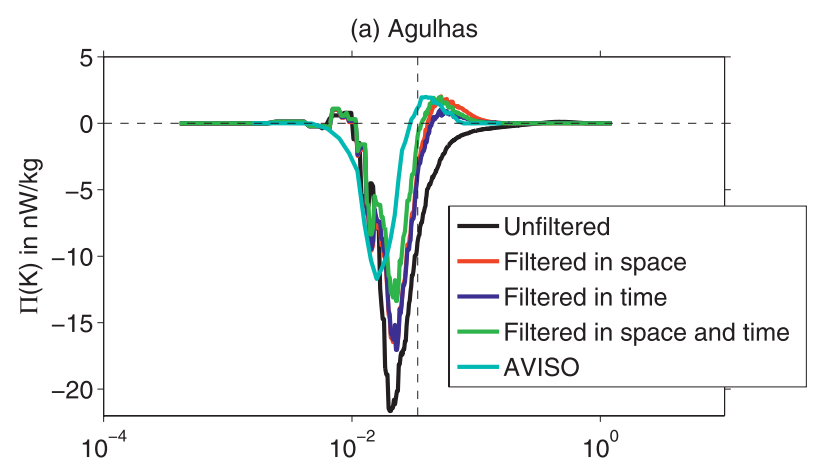

(b) Malvinas

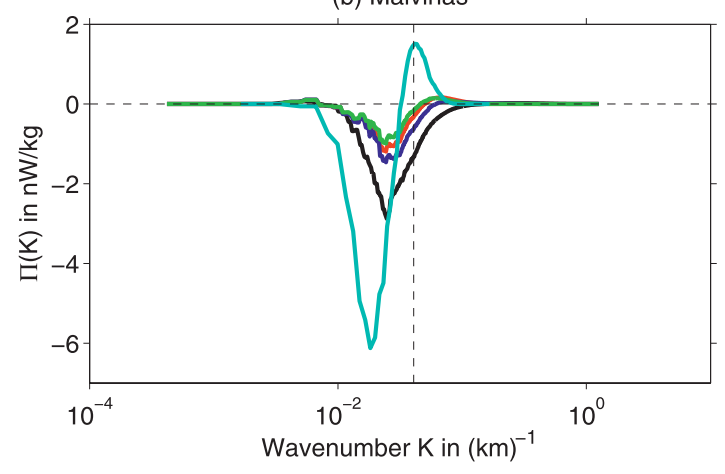

FIG. 9. As in Fig. 8, but for (a) Agulhas and (b) Malvinas.

the QG results with zero or low viscosity (i.e., black and cyan curves in Fig. 3).

Table 2 lists the estimated values of nondimensional $\nu$, and of FtoI (the ratio of peak forward to peak inverse fluxes), for the six regions of NLOM output. Recall that nondimensional $\nu$ in the two-layer QG model equals the dimensional viscosity divided by the product of $L_{d}$ and the mean shear $\left(\bar{u}_{1}-\bar{u}_{2}\right)$. The NLOM dimensional $\nu$ value was given in section $4 a$. For each of the six regions, the deformation radius $L_{d}$ is obtained from the Chelton et al. (1998) dataset as described above. To estimate the NLOM mean shear (the NLOM equivalent of $\bar{u}_{1}-\bar{u}_{2}$ in the two-layer QG model), we computed the time-averaged velocity components $\bar{u}_{1}, \bar{u}_{6}, \bar{v}_{1}$, and $\bar{v}_{6}$ at all model gridpoints from year 2003 of the NLOM simulation, where here subscripts 1 and 6 respectively denote the top and bottom layers of the six layers in NLOM. ${ }^{3}$ Then over each of our six regions of interest, we computed the area-averaged RMS shear as

\footnotetext{
${ }^{3}$ Top- and bottom-layer NLOM velocities were not saved over all three of the years 2001-03 analyzed in this paper. Note that we are analyzing NLOM runs performed some time ago, for purposes other than those envisioned for the present paper.
} 
(a) Gulf Stream

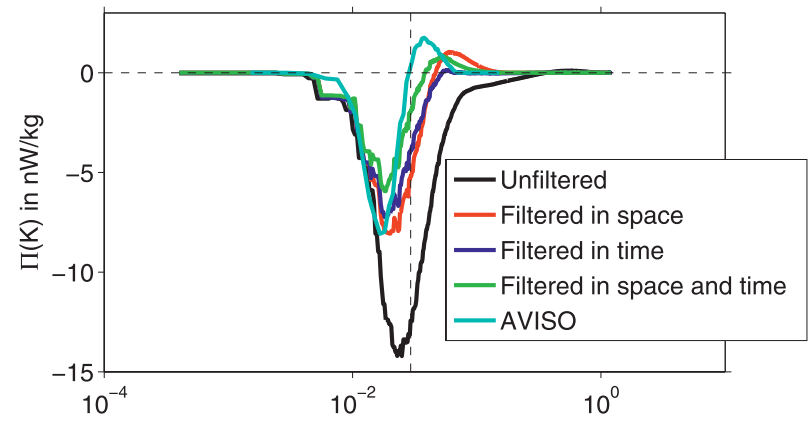

(b) Kuroshio

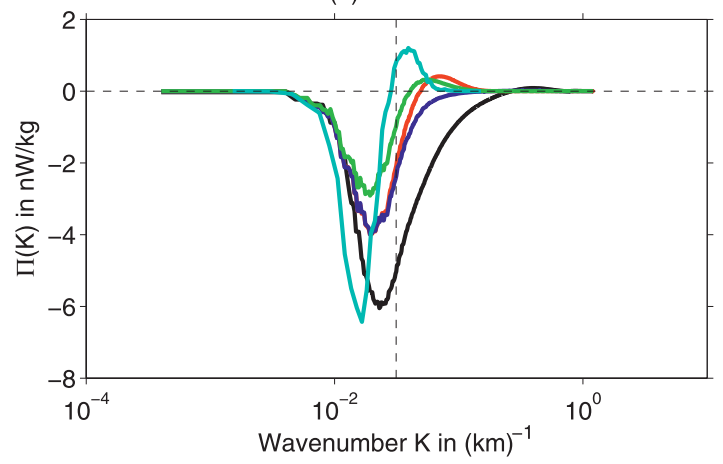

FIG. 10. As in Fig. 8, but for (a) Gulf Stream and (b) Kuroshio.

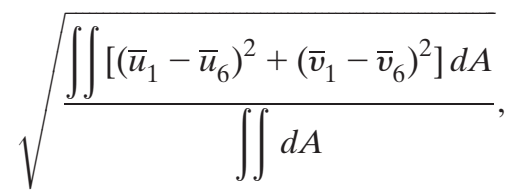

where $d A$ represents an element of area. Table 2 demonstrates that the NLOM nondimensional $\nu$ values are of order 0.01, suggesting that our earlier estimates of $\nu=$ 0.1 for the ocean may have been too large. Table 2 shows that the FtoI values generally decrease with decreasing values of nondimensional $\nu$, in qualitative agreement with the QG results. To make a more quantitative comparison, we note that the $\nu=0.01 \mathrm{QG}$ experiment listed in Table 1 has an FtoI value of 0.034, which is of the same order as the FtoI values in the first four regions listed in Table 2, all of which have nondimensional $\nu$ values of the same order as 0.01 .

Figures 8-10 also display the spectral fluxes computed from NLOM output that has been filtered in space, time, or both space and time, in an attempt to mimic the filtering inherent in the construction of AVISO gridded altimeter data. As in the results computed from filtered output of the idealized model, the filters act on model output, not on the model itself as it is running. We choose to use Blackman filters in both space and time. Since the AVISO product is put out every seven days,
TABLE 2. Computed nondimensional viscosities $\nu$ and FtoI values (see text) for six regions of NLOM output. Results listed in order of descending $\nu$.

\begin{tabular}{lcl}
\hline \multicolumn{1}{c}{ Region } & Nondimensional $\nu$ & \multicolumn{1}{c}{ FtoI } \\
\hline Midlatitude southeast Pacific & 0.015 & 0.050 \\
High-latitude southeast Pacific & 0.011 & 0.024 \\
Malvinas & 0.0076 & 0.0076 \\
Kuroshio & 0.0039 & 0.014 \\
Gulf Stream & 0.0038 & 0.0071 \\
Agulhas & 0.0029 & 0.0050 \\
\hline
\end{tabular}

on a $1 / 3^{\circ}$ grid, we choose a temporal Blackman filter which goes to zero seven days from the central time, and a spatial Blackman filter which goes to zero at a distance equal to $37 \mathrm{~km}\left(1 / 3^{\circ}\right.$ of latitude) from the central gridpoint. Thus the chosen Blackman filters have length and time scales comparable to those used in constructing the AVISO product, although the analogy is of course rough. The effect on the computed spectral fluxes is similar to the effect seen in the fluxes computed from filtered versions of the QG model output. Filtering in space again increases the estimated forward cascade, even in cases where there was little forward cascade in the unfiltered realistic model output. Again we see the possibility that the forward cascade shown in Scott and Wang (2005) may be an artifact of the relatively low resolution of present-day gridded altimeter data. Filtering in either space or time diminishes the strength of the inverse cascade, and filtering in both further diminishes the inverse cascade. Filtering in either space or time also tends to shift the zero-crossings of the spectral flux curves toward lower wavenumbers.

Figures 8-10 include the AVISO spectral fluxes, which share some characteristics of the filtered NLOM results. In general the AVISO forward fluxes are substantially larger than the forward fluxes in their unfiltered NLOM counterparts. The AVISO FtoI values range from 0.17 to 0.31 over the six regions. The AVISO fluxes are shifted toward lower wavenumbers relative to the fluxes computed from unfiltered NLOM output. Finally, in contrast to the unfiltered NLOM results, the zero crossings of the AVISO fluxes lie at wavenumbers equal to or lower than the deformation wavenumber.

We compare the strength of the inverse cascade in AVISO versus NLOM by computing the ratio of the minimum of the negative lobe at larger scales in the AVISO $\Pi_{\mathrm{KE}, 1}(K)$ curve to the minimum negative $\Pi_{\mathrm{KE}, 1}(K)$ value at larger scales computed from unfiltered NLOM output. This ratio is 0.14 in the high-latitude Southeast Pacific, 1.1 in the Kuroshio, 2.1 in the Malvinas, and between $0.4-0.6$ in the other three regions. This order of magnitude agreement in five of the six regions is encouraging considering that the minimum value of the 
negative lobes changes by two orders of magnitude across the six regions. The order of magnitude agreement bolsters the claims of Scott and Wang (2005) that the inverse cascade at larger scales is a robust feature of the oceanic general circulation.

The computed spectral fluxes are sensitive to the choice of spatial windows. The subject of windows for spectral computations in nonperiodic data has received a great deal of scrutiny over the years (e.g., Priestley 1981). The window utilized here, constucted from 9 overlapping Hanning windows in each horizontal direction, is close to unity over most of the domain and drops smoothly to zero at the edges. We also experimented with a Tukey window having a ratio of taper to constant sections equal to 0.2. The spectral fluxes computed from NLOM with the Tukey window (not shown here) are very nearly equal to those computed from the 9 overlapping Hanning windows employed in this paper. Spectral fluxes computed from coarser-resolution AVISO output are more sensitive to the usage of Tukey versus overlapping Hanning windows than are fluxes computed from NLOM output; AVISO fluxes can differ by up to a factor of about 2 between the two cases, for some wavenumbers. However, even in the AVISO fluxes the wavenumbers at which forward cascades are seen, and at which inverse cascades are seen, are virtually identical. Therefore none of our qualitative conclusions about the AVISO spectral fluxes, or our quantitative conclusions about the NLOM spectral fluxes, appear to be overly sensitive to the choice of spatial window, as long as the window lies near one over most of the domain.

\section{Summary and discussion}

In this paper we have examined the effects of horizontal eddy viscosity and of horizontal grid resolution on geostrophic turbulence, with a particular focus on the spectral fluxes $\Pi_{\mathrm{KE}, 1}(K)$ of surface ocean geostrophic kinetic energy in the isotropic wavenumber $(K)$ domain. We have utilized outputs from idealized two-layer quasigeostrophic (QG) turbulence model simulations and realistic global numerical model (NLOM) simulations in this endeavor, and compared the model spectral fluxes to fluxes computed from present-day gridded satellite altimeter products (AVISO). We are motivated by considerations of ocean energetics and by the widespread use of horizontal eddy viscosity in models. The addition of viscosity to the QG model yields a forward energy cascade at length scales near the deformation radius toward smaller scales, as is apparently seen in computations of ocean surface spectral kinetic energy fluxes computed from AVISO data. Viscosity arguably represents the physics of mesoscale coupling to submesoscale motions and internal waves. Viscosity can dissipate a substantial amount of energy in the QG model. A sensitivity study in section 3 of this paper indicates that viscosity impacts the statistics of eddies in QG turbulence models, but that the model statistics can lie near observed ranges as long as the viscosity is not too large.

Insufficient horizontal resolution-be it the resolution chosen to run the QG simulations at, or a deliberate filtering in space and/or time to downgrade the resolution of the QG or realistic ocean model output after the simulation is completed - can also yield a forward cascade at smaller scales. This filtering mimics the filtering of along-track satellite altimeter data onto the AVISO grids. It is therefore difficult to tell whether the forward spectral kinetic energy fluxes observed for small scales in the Scott and Wang (2005) altimeter-based calculations of $\Pi_{\mathrm{KE}, 1}(K)$ are physical or an artifact of the smoothing inherent in constructing the AVISO gridded altimeter product.

Similar conclusions were reached by Boer and Shepherd (1983) in their pioneering computation of spectral kinetic energy fluxes in atmospheric data. T. Shepherd 2010 (personal communication) notes that "explicit calculation of nonlinear interactions is inherently limited by the resolution of the data set, especially close to the resolution limit." Boer and Shepherd's (1983) attempt to account for missing nonlinear interactions at the limiting resolutions of their dataset substantially reduced the estimated forward energy flux at their highest wavenumbers (see Fig. 13a of their paper). In addition, Shepherd (1987) found that the crossover from negative to positive flux occurs at different wavenumbers for different truncations of atmospheric datasets, such that the forward flux is not a reliable feature. A simple interpretation of the limiting effect of spatial resolution was given in the 2010 personal communication with Shepherd: "Following Fjortoft (1953), when computing triads over a finite spectral range, energy must cascade both upscale and downscale and so an apparent downscale cascade is inevitable in such a calculation even if there is no such cascade in nature. It's also obvious that the enstrophy flux cannot really go to zero at the truncation wavenumber; this too points to the fact that resolved fluxes are strongly affected by truncation."

The proposed wide-swath satellite altimeter (Fu and Ferrari 2008; Fu et al. 2012) will map sea surface heights at much higher horizontal resolution than is currently possible. Energy cascades to small scales ultimately lead to mixing. Mixing in the upper ocean has a greater impact on the oceanic meridional overturning circulation than mixing in the abyssal ocean (Scott and Marotzke 2002). This adds importance to the quest to understand whether the forward fluxes seen in present-generation 
altimeter data represent real physics or sampling artifacts. Sea surface height measurements can only be used to constrain geostrophic velocities. Therefore, even data from a wide-swath altimeter can only be used to compute spectral fluxes arising from the geostrophic part of the flow. However, wide-swath altimeter measurements of sea surface height at small scales will be able to constrain the height fields of models at small scales, and from such models (e.g., Capet et al. 2008) the spectral fluxes arising from ageostrophic flows can be computed.

We have shown that the maximum strength of the inverse kinetic energy cascade [i.e. the minimum of the negative lobes in the spectral fluxes $\left.\Pi_{\mathrm{KE}, 1}(K)\right]$ computed from realistic models and altimetry generally agree to within a factor of about 2, even as the spectral flux itself varies by two orders of magnitude over the six oceanic regions studied here. As far as we know, this is the first direct comparison of inverse cascades computed in realistic ocean circulation models versus altimeter data. In both the QG and realistic models, the existence of the negative spectral fluxes (inverse cascades) at larger scales seen in current-generation altimeter data is robust to the filtering exercise, but the exact strength of the inverse cascades is not robust to filtering. The planned wide-swath altimeter should therefore aid in refining estimates of the strength of the oceanic inverse cascade as well as constraining dynamics at smaller scales.

Acknowledgments. We acknowledge two anonymous reviewers whose comments led to substantial improvements and clarifications in this manuscript. BKA thanks Detlef Stammer for hosting the workshop "Below the Rossby radius: Workshop on small-scale variability in the general circulation of the atmosphere and oceans," which provided added inspiration for this study. We acknowledge helpful discussions with Dudley Chelton, Joe LaCasce, Roger Samelson, Ted Shepherd, and Alan Wallcraft. BKA received support from Office of Naval Research Grant N00014-11-1-0487, National Science Foundation (NSF) Grants OCE-0924481 and OCE09607820, and University of Michigan startup funds. KLP acknowledges support from Woods Hole Oceanographic Institution bridge support funds. RBS acknowledges support from NSF grants OCE-0960834 and OCE-0851457, a contract with the National Oceanography Centre, Southampton, and a NASA subcontract to Boston University. JFS and JGR were supported by the projects "Global and remote littoral forcing in global ocean models" and "Agesotrophic vorticity dynamics of the ocean," respectively, both sponsored by the Office of Naval Research under program element
601153N. The Naval Research Laboratory contribution has been approved for public release.

\section{REFERENCES}

Arbic, B. K., and G. R. Flierl, 2004: Baroclinically unstable geostrophic turbulence in the limits of strong and weak bottom Ekman friction: Application to mid-ocean eddies. J. Phys. Oceanogr., 34, 2257-2273.

, and R. B. Scott, 2008: On quadratic bottom drag, geostrophic turbulence, and oceanic mesoscale eddies. J. Phys. Oceanogr., 38, 84-103.

—, G. R. Flierl, and R. B. Scott, 2007: Cascade inequalities for forced-dissipated geostrophic turbulence. J. Phys. Oceanogr., 37, 1470-1487.

, and Coauthors, 2009: Estimates of bottom flows and bottom boundary layer dissipation of the oceanic general circulation from global high-resolution models. J. Geophys. Res., 114, C02024, doi:10.1029/2008JC005072.

Boer, G. J., and T. G. Shepherd, 1983: Large-scale two-dimensional turbulence in the atmosphere. J. Atmos. Sci., 40, 164-184.

Brown, E. D., and W. B. Owens, 1981: Observations of the interactions between the internal wave field and the mesoscale flow. J. Phys. Oceanogr., 11, 1474-1480.

Bühler, O., and M. E. McIntyre, 2005: Wave capture and wavevortex duality. J. Fluid Mech., 534, 67-95.

Capet, X., J. C. McWilliams, M. J. Molemaker, and A. F. Shchepetkin, 2008: Mesoscale to submesoscale transition in the California current system. Part III: Energy balance and flux. J. Phys. Oceanogr., 38, 2256-2269.

Chelton, D. B., R. A. deSzoeke, M. G. Schlax, K. El Naggar, and N. Siwertz, 1998: Geographical variability of the first-baroclinic Rossby radius of deformation. J. Phys. Oceanogr., 28, 433-460.

_ J. C. Ries, B. J. Haines, L.-L. Fu, and P. S. Callahan, 2001: Satellite altimetry. Satellite Altimetry and Earth Sciences, L.-L. Fu and A. Cazenave, Eds., Academic Press, 1-131.

_ M. G. Schlax, and R. M. Samelson, 2011: Global observations of nonlinear mesoscale eddies. Prog. Oceanogr., 91, 167-216.

D'Asaro, E., C. Lee, L. Rainville, R. Harcourt, and L. Thomas, 2011: Enhanced turbulence and energy dissipation at ocean fronts. Science, 332, 318-322, doi:10.1126/science.1201515.

Dewar, W. K., and A. McC. Hogg, 2010: Topographic inviscid dissipation of balanced flow. Ocean Modell., 32, 1-13, doi:10.1016/j.ocemod.2009.03.007.

Ducet, N., P.-Y. Le Traon, and G. Reverdin, 2000: Global highresolution mapping of ocean circulation from TOPEX/Poseidon and ERS-1 and -2. J. Geophys. Res., 105, 19 477-19 498.

Fjortoft, R., 1953: On the changes in the spectral distributions of kinetic energy for two-dimensional non-divergent flow. Tellus, $\mathbf{5}, 225-230$.

Flierl, G. R., 1978: Models of vertical structure and the calibration of two-layer models. Dyn. Atmos. Oceans, 2, 341-381.

Frisch, U., 1995: Turbulence, the Legacy of A.N. Kolmolgorov. Cambridge University Press, 296 pp.

Fu, L.-L., and G. R. Flierl, 1980: Nonlinear energy and enstrophy transfers in a realistically stratified ocean. Dyn. Atmos. Oceans, 4, 219-246.

—_, and R. Ferrari, 2008: Observing oceanic submesoscale processes from space. Eos, Trans. Amer. Geophys. Union, 89, 488, doi:10.1029/2008EO480003.

, D. Alsdorf, R. Morrow, E. Rodriguez, and N. Mognard, Eds., 2012: SWOT: The surface water and ocean topography mission: 
Wide-swath altimetric measurement of water elevation on earth. Jet Propulsion Laboratory JPL-Publ. 12-05, 228 pp.

Hurlburt, H. E., and J. D. Thompson, 1980: A numerical study of Loop Current intrusions and eddy shedding. J. Phys. Oceanogr., 10, 1611-1651.

Jochum, M., G. Danabasoglu, M. Holland, Y.-O. Kwon, and W. G. Large, 2008: Ocean viscosity and climate. J. Geophys. Res., 113, C06017, doi:10.1029/2007JC004515.

LaCasce, J. H., 1996: Baroclinic vortices over a sloping bottom. Ph.D. dissertation, Massachusetts Institute of TechnologyWoods Hole Oceanographic Institution Joint Program, $220 \mathrm{pp}$

_ 2012: Surface quasi-geostrophic solutions and baroclinic modes with exponential stratification. J. Phys. Oceanogr., 42, $569-580$.

Lapeyre, G., 2009: What vertical mode does the altimeter reflect? On the decomposition in baroclinic modes and a surfacetrapped mode. J. Phys. Oceanogr., 39, 2857-2874.

Larichev, V., and G. Reznik, 1976a: Strongly nonlinear two-dimensional isolated Rossby waves. Oceanologia, 16, 547-550.

_ and - 1976b: Two-dimensional Rossby soliton: An exact solution. Rep. USSR Acad. Sci., 231, 1077-1079.

— mogeneous model of fully developed baroclinic instability. J. Phys. Oceanogr., 25, 2285-2297.

Le Traon, P.-Y., F. Nadal, and N. Ducet, 1998: An improved mapping method of multi-satellite altimeter data. J. Atmos. Oceanic Technol., 15, 522-534.

Marshall, D. P., and A. C. Naveira-Garabato, 2008: A conjecture on the role of bottom-enhanced diapycnal mixing in the parameterization of geostrophic eddies. J. Phys. Oceanogr., 38, $1607-1613$.

McWilliams, J. C., 1984: The emergence of isolated coherent vortices in turbulent flow. J. Fluid Mech., 146, 21-43.

Müller, P., 1976: On the diffusion of momentum and mass by internal gravity waves. J. Fluid Mech., 77, 789-823.

_- 1979: A note on the variability of eddy diffusion coefficients in the ocean. Dyn. Atmos. Oceans, 3, 267-274.

— and D. Olbers, 1975: On the dynamics of internal waves in the deep ocean. J. Geophys. Res., 80, 3848-3860.

Munk, W., and C. Wunsch, 1998: Abyssal recipes II: Energetics of tidal and wind mixing. Deep-Sea Res. I, 45, 1977-2010.

Nikurashin, M., and R. Ferrari, 2011: Global energy conversion rate from geostrophic flows into internal lee waves in the deep ocean. Geophys. Res. Lett., 38, L08610, doi:10.1029/2011GL046576.

Polzin, K. L., 2008: Mesoscale eddy-internal wave coupling. Part I: Symmetry, wave capture, and results from the mid-ocean dynamics experiment. J. Phys. Oceanogr., 38, 2556-2574.

_ 2010: Mesoscale eddy-internal wave coupling. Part II: Energetics and results from POLYMODE. J. Phys. Oceanogr., 40, 789-801.

Priestley, M. B., 1981: Spectral Analysis and Time Series. Academic Press, $890 \mathrm{pp}$.

Richman, J. G., C. Wunsch, and N. G. Hogg, 1977: Space and time scales of mesoscale motion in the Western North Atlantic. Rev. Geophys. Space Phys., 15, 385-420.

Schlösser, F., and C. Eden, 2007: Diagnosing the energy cascade in a model of the North Atlantic. Geophys. Res. Lett., 34, L02604, doi:10.1029/2006GL027813.

Schmitz, W. J., 1996: On the world ocean circulation. Vol. I: Some global features/North Atlantic circulation. Woods Hole Oceanographic Institution Tech. Rep. WHOI-96-03, 140 pp.
Scott, J. R., and J. Marotzke, 2002: The location of diapycnal mixing and the meridional overturning circulation. J. Phys. Oceanogr., 32, 3578-3595.

Scott, R. B., 1999: Mechanical energy flux to the surface geostrophic flow using TOPEX/Poseidon data. Phys. Chem. Earth, 24, 399-402.

— , and F. Wang, 2005: Direct evidence of an oceanic inverse kinetic energy cascade from satellite altimetry. J. Phys. Oceanogr., 35, 1650-1666.

— turbulence: Implications for ocean energetics. J. Phys. Oceanogr., 37, 673-688.

— surface geostrophic flow of the World Ocean. Deep-Sea Res. I, 50, 295-304.

_ J. A. Goff, A. C. N. Garabato, and A. J. G. Nurser, 2011: Global rate and spectral characteristics of internal gravity wave generation by geostrophic flow over topography. J. Geophys. Res., 116, C09029, doi:10.1029/2011JC007005.

Sen, A., R. B. Scott, and B. K. Arbic, 2008: Global energy dissipation rate of deep-ocean low-frequency flows by quadratic bottom boundary layer drag: Computations from current-meter data. Geophys. Res. Lett., 35, L09606, doi:10.1029/2008GL033407.

Shepherd, T. G., 1987: Inhomogeneous two-dimensional turbulence in the atmosphere. Advances in Turbulence, G. ComteBellot and J. Mathieu, Eds., Springer-Verlag, 269-278.

Shriver, J. F., H. E. Hurlburt, O. M. Smedstad, A. J. Wallcraft, and R. C. Rhodes, $2007: 1 / 32^{\circ}$ real-time global ocean prediction and value-added over $1 / 16^{\circ}$ resolution. J. Mar. Syst., 65, 3-26.

Stammer, D., 1997: Global characteristics of ocean variability estimated from regional TOPEX/Poseidon altimeter measurements. J. Phys. Oceanogr., 27, 1743-1769.

Stern, M. E., 1975: Minimal properties of planetary eddies. J. Mar. Res., 33, 1-13.

Thompson, A. F., and W. R. Young, 2006: Scaling baroclinic eddy fluxes: Vortices and energy balance. J. Phys. Oceanogr., 36, $720-738$.

— and - 2007: Baroclinic eddy heat fluxes: Zonal flows and energy balance. J. Atmos. Sci., 64, 3214-3231.

Tulloch, R., J. Marshall, C. Hill, and K. S. Smith, 2011: Scales, growth rates, and spectral fluxes of baroclinic instability in the ocean. J. Phys. Oceanogr., 41, 1057-1076.

Vallis, G. K., 2006: Atmospheric and Oceanic Fluid Dynamics: Fundamentals and Large-Scale Circulation. Cambridge University Press, 745 pp.

Wallcraft, A. J., A. B. Kara, H. E. Hurlburt, and P. A. Rochford, 2003: The NRL Layered Ocean Model (NLOM) with an embedded mixed layer sub-model: Formulation and tuning. J. Atmos. Oceanic Technol., 20,1601-1615.

,$- \ldots$, and $—, 2005$ : Convergence of Laplacian diffusion versus resolution of an ocean model. Geophys. Res. Lett., 32, L07604, doi:10.1029/2005GL022514.

Wright, C., R. B. Scott, D. G. Furnival, P. Alliot, and F. Vermet, 2013: Global observations of ocean-bottom subinertial current dissipation. J. Phys. Oceanogr., 43, 402-417.

Wunsch, C., 1997: The vertical partition of oceanic horizontal kinetic energy. J. Phys. Oceanogr., 27, 1770-1794.

_ 1998: The work done by the wind on the oceanic general circulation. J. Phys. Oceanogr., 28, 2332-2340.

_ 2001: Ocean observations and the climate forecast problem. Meteorology at the Millenium, R. P. Pearce, Ed., Academic Press, 217-224. 